Article

\title{
Skew Continuous Morphisms of Ordered Lattice Ringoids
}

\author{
Sergey Victor Ludkowski
}

Department of Applied Mathematics, Moscow State Technical University MIREA, avenue Vernadsky 78, Moscow 119454, Russia; Ludkowski@mirea.ru; Tel.: +7-495-434-7565

Academic Editor: Hvedri Inassaridze

Received: 8 November 2015; Accepted: 24 February 2016; Published: 16 March 2016

\begin{abstract}
Skew continuous morphisms of ordered lattice semirings and ringoids are studied. Different associative semirings and non-associative ringoids are considered. Theorems about properties of skew morphisms are proved. Examples are given. One of the main similarities between them is related to cones in algebras of non locally compact groups.
\end{abstract}

Keywords: non-associative; algebra; morphism; idempotent; skew; semiring; ringoid

Mathematics Subject Classification 2010: 08A99; 16Y60; 17A01; 17D99; 22A26; 22A30

\section{Introduction}

Semirings, ringoids, algebroids and non-associative algebras play important role in algebra and among them ordered semirings and lattices as well [1-8]. This is also motivated by idempotent mathematical physics naturally appearing in quantum mechanics and quantum field theory (see, for example, [9] and references therein). They also arise from the consideration of algebroids and ringoids associated with non locally compact groups. Namely, this appears while the studies of representations of non locally compact groups, quasi-invariant measures on them and convolution algebras of functions and measures on them [10-13]. The background for this is A. Weil's theorem (see [14]) asserting that if a topological group has a quasi-invariant $\sigma$-additive non trivial measure relative to the entire group, then it is locally compact. Therefore, it appears natural to study inverse mapping systems of non locally compact groups and their dense subgroups. Such spectra lead to structures of algebroids and ringoids. Investigations of such objects are also important for making advances in representation theory of non locally compact groups.

In this paper methods of categorial topology are used (see [15-18] and references therein).

This article is devoted to ordered ringoids and semirings with an additional lattice structure. Their continuous morphisms are investigated in Section 3. Preliminaries are given in Section 2. Necessary definitions 2.1-2.4 are recalled. For a topological ringoid $K$ and a completely regular topological space $X$ new ringoids $C(X, K)$ are studied, where $C(X, K)$ consists of all continuous mappings $f: X \rightarrow K$ with point-wise algebraic operations. Their ideals, topological directed structures and idempotent operations are considered in Lemmas 2.6, 2.8, 2.9, 2.12 and Corollary 2.7. There are also given several examples $2.13-2.18$ of objects. One of the main examples between them is related to cones in algebras of non locally compact groups. Another example is based on ordinals. Construction of ringoids with the help of inductive limits is also considered.

Structure and properties of these objects are described in Section 3. Definitions of morphisms of ordered semirings and some their preliminaries are described in Subsection 3.1. An existence of idempotent $K$-homogeneous morphisms under definite conditions is proved in Lemma 3.4. A relation between order preserving weakly additive morphisms and non-expanding morphisms is given in 
Lemma 3.7. An extension of an order preserving weakly additive morphism is considered in Lemma 3.9.

Then a weak ${ }^{*}$ topology on a family $\mathcal{O}(X, K)$ of all order preserving weakly additive morphisms on a Hausdorff topological space $X$ with values in $K$ is taken. The weak ${ }^{*}$ compactness of $\mathcal{O}(X, K)$ under definite conditions is proved in Theorem 3.10. Further in Proposition 3.11 there is proved that $I(X, K)$ and $I_{h}(X, K)$ are closed in $\mathcal{O}(X, K)$, where $I(X, K)$ denotes the set of all idempotent $K$-valued morphisms, also $I_{h}(X, K)$ denotes its subset of idempotent homogeneous morphisms.

Categories related to morphisms and ringoids are presented in Subsection 3.2. An existence of covariant functors, their ranges and continuity of morphisms are studied in Lemmas 3.14, 3.16, 3.21, 3.34 and Propositions 3.15, 3.22. In Propositions 3.24, 3.26 and 3.29 such properties of functors as being monomorphic and epimorphic are investigated. Supports of functors are studied in Proposition 3.31. Moreover, in Proposition 3.32 it is proved that definite functors preserve intersections of closed subsets. Then functors for inverse systems are described in Proposition 3.33. Bi-functors preserving pre-images are considered in Proposition 3.35. Monads in certain categories are investigated in Theorem 3.38. Exact sequences in categories are considered in Proposition 3.39.

Lattices associated with actions of groupoids on topological spaces are investigated in subsection 3.3. Supports of $(T, G)$-invariant semi-idempotent continuous morphisms are estimated in Proposition 3.42, where $G$ is a topological groupoid and $T$ is its representation described in Lemma 3.40. Structures of families of all semi-idempotent continuous morphisms associated with a groupoid $G$ and a ringoid $K$ are investigated in Proposition 3.43 and Theorems 3.44, 3.45.

The main results are Propositions 3.22, 3.24, 3.29, 3.32, 3.33, 3.35, 3.39, 3.43, Theorems 3.38, 3.44 and 3.45. All main results of this paper are obtained for the first time. The obtained results can be used for further studies of such objects, their classes and classification. They can be applied to investigations of non locally compact group algebras also.

\section{Ringoids and Lattice Structure}

\subsection{Preliminaries}

To avoid misunderstandings we first present our definitions.

1. Definitions. Let $K$ be a set and let two operations $+: K^{2} \rightarrow K$ the addition and $\times: K^{2} \rightarrow K$ the multiplication be given so that $K$ is a semigroup (with associative binary operations) or a quasigroup (with may be non-associative binary operations) relative to + and $\times$ with neutral elements $e_{+}=: 0$ and $e_{\times}=: 1$ so that $a \times 0=0 \times a=0$ for each $a \in K$ and either the right distributivity $a(b+c)=a b+a c$ for every $a, b, c \in K$ or the left distributivity $(b+c) a=b a+c a$ for every $a, b, c \in K$ is accomplished, then $K$ is called a semiring or a ringoid respectively with either right or left distributivity correspondingly. If it is simultaneously right and left distributive, then it is called simply a semiring or a ringoid respectively.

A semiring $K$ (or a ringoid, or a ring, or a non-associative ring) having also a structure of a linear space over a field $\mathbf{F}$ and such that $\alpha(a+b)=\alpha a+\alpha b, 1 a=a, \alpha(a b)=(\alpha a) b=a(\alpha b)$ and $(\alpha \beta) a=\alpha(\beta a)$ for each $\alpha, \beta \in \mathbf{F}$ and $a, b \in K$ is called a semialgebra (or an algebroid, or an algebra or a non-associative algebra correspondingly).

A semiring $K$ (or a semialgebra and so on) supplied with a topology on $K$ (or on $K$ and F correspondingly) relative to which algebraic operations are continuous is called a topological semiring (or a topological semialgebra and so forth correspondingly).

A set $K$ with binary operations $\mu_{1}, \ldots, \mu_{n}$ will also be called an algebraic object. An algebraic object is commutative relative to an operation $\mu_{p}$ if $\mu_{p}(a, b)=\mu_{p}(b, a)$ for each $a, b \in K$.

An algebraic object $K$ with binary operations $\mu_{1}, \ldots, \mu_{n}$ is called either directed or linearly ordered or well-ordered if it is such as a set correspondingly and its binary operations preserve an ordering: $\mu_{p}(a, b) \leq \mu_{p}(c, d)$ for each $p=1, \ldots, n$ and for every $a, b, c, d \in K$ so that $a \leq c$ and $b \leq d$ when $a, b, c, d$ belong to the same linearly ordered set $Z$ in $K$. 
Henceforward, we suppose that the minimal element in an ordered $K$ is zero.

Henceforth, for semialgebras, non-associative algebras or algebroids $A$ speaking about ordering on them we mean that only their non-negative cones $K=\{y: y \in A, 0 \leq y\}$ are considered. For non-negative cones $K$ in semialgebras, non-associative algebras or algebroids only the case over the real field will be considered.

2. Definition. A (non-associative) topological algebra or a topological ringoid, etc., we call topologically simple if it does not contain closed ideals different from $\{0\}$ and $K$, where $K \neq\{0\}$.

3. Definition. We consider a directed set $K$ which satisfies the condition:

$(D W)$ for each linearly ordered subset $A$ in $K$ there exists a well-ordered subset $B$ in $K$ such that $A \subset B$.

4. Definitions. Let $K$ be a well-ordered (or directed satisfying condition $3(D W)$ ) either semiring or ringoid (or a non-negative cone in a algebroid over the real field $\mathbf{R}$ ) such that

(1) $\sup E \in K$ for each $E \in T$, where $T$ is a family of subsets of $K$.

If $K$ is a directed topological either semiring or ringoid, we shall suppose that it is supplied with a topology

(2) $\tau=\tau_{K}$ so that every set

$L_{b}:=\{y: y \in K, y<b$ or $y$ is not comparable with $b\}$ and

$G_{b}:=\{y: y \in K, b<y$ or $y$ is not comparable with $b\}$

is open relative to it.

That is, if a set $Z$ is linearly ordered in $K$ this topology $\tau_{K}$ provides the hereditary topology on $Z$ which is not weaker than the interval topology on $Z$ generated by the base $\left\{(a, b)_{Z}: a<b \in Z\right\}$, where $(a, b)_{Z}:=\{c: c \in Z, a<c<b\}$.

For a completely regular topological space $X$ and a topological semiring (or ringoid) $K$ let $C(X, K)$ denote a semiring (or a ringoid respectively) of all continuous mappings $f: X \rightarrow K$ with the element-wise addition $(f+g)(x)=f(x)+g(x)$ and the element-wise multiplication $(f g)(x)=f(x) g(x)$ operations for every $f, g \in C(X, K)$ and $x \in X$.

If $K$ is a directed semiring (or a directed ringoid) and $X$ is a linearly ordered set, $C_{+}(X, K)$ (or $C_{-}(X, K)$ ) will denote the set of all monotone non-decreasing (or non-increasing correspondingly) maps $f \in C(X, K)$.

For the space $C(X, K)$ (or $C_{+}(X, K)$ or $\left.C_{-}(X, K)\right)$ we suppose that

(3) a family $T$ of subsets of $K$ contains the family $\{f(X): f \in C(X, K)\}$ (or $\left\{f(X): f \in C_{+}(X, K)\right\}$ or $\left\{f(X): f \in C_{-}(X, K)\right\}$ correspondingly) and $K$ satisfies Condition (1).

5. Remark. For example, the class $O n$ of all ordinals has the addition $\mu_{1}=+_{o}$ and the multiplication $\mu_{2}=\times_{o}$ operations which are generally non-commutative, associative, with unit elements 0 and 1 respectively, on $O n$ the right distributivity is satisfied (see Propositions 4.29-4.31 and Examples 1-3 in [19]). Relative to the interval topology generated by the base $\{(a, b): a<b \in O n\}$ the class $O n$ is the topological well-ordered semiring, where $(a, b)=\{c: c \in O n, a<c<b\}$. For each non-void set $A$ in $O n$ there exists sup $A \in O n$ (see [20]).

If $K$ is a linearly ordered non-commutative relative to the addition semiring (or a ringoid), then the new operation $(a, b) \mapsto \max (a, b)=: a+{ }_{2} b$ defines the commutative addition. Then $c\left(a+{ }_{2} b\right)=$ $\max (c a, c b)=c a+{ }_{2} c b$ and $\left(a+{ }_{2} b\right) c=\max (a c, b c)=a c+{ }_{2} b c$ for every $a, b, c \in K$, that is $(T,+2, \times)$ is left and right distributive.

As an example of a semiring (or a ringoid) $K$ in Definitions 4 one can take $K=O n$ or $K=\{A: A \in O n,|A| \leq b\}$, where $b$ is a cardinal number such that $\aleph_{0} \leq b$. Each segment $[a, b]:=\{c: c \in O n, a \leq c \leq b\}$ is compact in On, where $a<b \in O n$. Evidently, $K=O n$ satisfies Condition 4(1), since sup $E$ exists for each set $E$ in $O n$ (see [20]).

Particularly, if a topological space $X$ is compact and $C(X, K)$ is a semiring (or a ringoid) of all continuous mappings $f: X \rightarrow K$, then a family $T$ contains the family of compact subsets 
$\{f(X): f \in C(X, K)\}$, since a continuous image of a compact space is compact (see Theorem 3.1.10 [21]).

It is possible to modify Definition 4 in the following manner. For a well-ordered $K$ without Condition 4(1) one can take the family of all continuous bounded functions $f: X \rightarrow K$ and denote this family of functions by $C(X, K)$ for the uniformity of the notation.

For a directed $K$ satisfying Condition $3(D W)$ without Condition $4(1)$ it is possible to take the family of all monotone non-decreasing (or non-increasing) bounded functions $f: X \rightarrow K$ for a linearly ordered set $X$ and denote this family by $C_{+}(X, K)\left(C_{-}(X, K)\right.$ correspondingly) also.

Naturally, $C(X, K)$ has also the structure of the left and right module over the semiring (or the ringoid correspondingly) $K$, i.e., af and $f a$ belong to $C(X, K)$ for each $a \in K$ and $f \in C(X, K)$. To any element $a \in K$ the constant mapping $g^{a} \in C(X, K)$ corresponds such that $g^{a}(x)=a$ for each $x \in X$. If $K$ is right (or left) distributive, then $q(f+h)=q f+q h$ (or $(f+h) q=f q+h q$ correspondingly) for every $q, f, h \in C(X, K)$.

The semiring (or the ringoid) $C(X, K)$ will be considered directed:

(1) $f \leq g$ if and only if $f(x) \leq g(x)$ for each $x \in X$.

Indeed, if $f, h \in C(X, K)$, then $a=\sup (f(X)) \in K$ and $b=\sup (h(X)) \in K$ according to Condition 4(1). Then there exists $c \in K$ so that $a \leq c$ and $b \leq c$, consequently, $f \leq g^{c}$ and $h \leq g^{c}$. Thus for each $f, h \in C(X, K)$ there exists $q \in C(X, K)$ so that $f \leq q$ and $h \leq q$. From $a+b \leq c+d$ and $a b \leq c d$ for each $a \leq c$ and $b \leq d$ in $K$ it follows that $f+q \leq g+h$ and $f q \leq g h$ for each $f \leq g$ and $q \leq h$ in $C(X, K)$.

If $f \leq g$ and $f \neq g$ (i.e. $\exists x \in X f(x) \neq g(x)$ ), then we put $f<g$.

For a mapping $f \in C(X, K)$ its support $\operatorname{supp}(f)$ is defined as usually

(2) $\operatorname{supp}(f):=\operatorname{cl}_{X}\{x: x \in X, f(x) \neq 0\}$, where $c l_{X} A$ denotes the closure of $A$ in $X$ when $A \subset X$.

Henceforth, we consider cases, when

(3) a topology on $X$ is sufficiently fine so that functions separate points in $X$, i.e., for each $x \neq z$ in $X$ there exists $f$ in $C(X, K)$ (or $C_{-}(X, K)$ or $C_{+}(X, K)$ correspondingly) such that $f(x) \neq f(z)$.

The latter is always accomplished in the purely algebraic discrete case.

\subsection{Directed Ringoids $C(X, K)$ of Mappings}

6. Lemma. If $E$ is a closed subspace in a topological space $X$, then $C(X, K \mid E):=$ $\{f: f \in C(X, K), \operatorname{supp}(f) \subset E\}$ is an ideal in $C(X, K)$.

Proof. If $f \in C(X, K \mid E)$ and $g \in C(X, K)$, then $f(x) g(x)=0$ and $g(x) f(x)=0$ when $f(x)=0$, consequently, $\operatorname{supp}(f g)$ and $\operatorname{supp}(g f)$ are contained in $E$. Moreover, if $f, h \in C(X, K \mid E)$, then $\operatorname{supp}(f+h)$ and $\operatorname{supp}(h+f)$ are contained in $E$, since $f(x)+h(x)=0$ and $h(x)+f(x)=0$ for each $x \in X \backslash E$, while $X \backslash E$ is open in $X$. Thus $C(X, K \mid E)$ is a semiring (or a ringoid respectively) and $C(X, K \mid E) C(X, K) \subseteq C(X, K \mid E)$ and $C(X, K) C(X, K \mid E) \subseteq C(X, K \mid E)$.

7. Corollary. If $E$ is clopen (i.e. closed and open simultaneously) in $X$, then $C(E, K)$ is an ideal in $C(X, K)$.

Proof. For a clopen topological subspace $E$ in $X$ one gets $C(E, K)$ isomorphic with $C(X, K \mid E)$, since each $f \in C(E, K)$ has the zero extension on $X \backslash E$.

8. Lemma. For a linearly ordered set $X$ and a directed semiring (ringoid) $K$ there are directed semirings (or ringoids correspondingly) $C_{+}(X, K)$ and $C_{-}(X, K)$.

Proof. The sets $C_{+}(X, K)$ and $C_{-}(X, K)$ are directed according to Condition 5(1) with a partial ordering inherited from $C(X, K)$. Since $a+b \leq c+d$ and $a b \leq c d$ for each $a \leq c$ and $b \leq d$ in $K$, then $f+q \leq g+h$ and $f q \leq g h$ for each $f \leq g$ and $q \leq h$ all either in $C_{+}(X, K)$ or $C_{-}(X, K)$. On the other hand, for each $f, h \in C(X, K)$ there exists $g^{c} \in C(X, K)$ so that $f \leq g^{c}$ and $h \leq g^{c}$ 
(see § 5). If $f(x) \leq f(y)$ and $h(x) \leq h(y)$ for $f, h \in C_{+}(X, K)$ and each $x \leq y$ in $K$, then $f(x)+h(x) \leq$ $f(y)+h(y)$ and $f(x) h(x) \leq f(y) h(y)$, consequently, $f+h$ and $f h$ are in $C_{+}(X, K)$. Analogously, if $f, h \in C_{-}(X, K)$, then $f+h$ and $f h$ are in $C_{-}(X, K)$. But a constant mapping $g^{c}$ belongs to $C_{+}(X, K)$ and $C_{-}(X, K)$. Thus $C_{+}(X, K)$ and $C_{-}(X, K)$ are directed semirings (or ringoids correspondingly).

9. Lemma. If $H=H_{X}$ is a covering of $X$ and $\tau_{K}$ is a topology on $K$ satisfying Conditions $3(D W)$ and $4(1-3)$, then a semiring (or ringoid or a non-negative cone in a algebroid over $\mathbf{R}) C(X, K)$ can be supplied with a topology relative to which it is a topological directed (TD) semiring (or a TD ringoid or a TD algebroid respectively).

Proof. Take a topology $\tau_{C}$ on $C(X, K)$ with the base $\beta_{C}$ formed by the following sets and their finite intersections:

(1) $B_{1}(g, A, V):=\{f: f \in C(X, K), f(A) \subset g+V\}$,

$B_{2}(g, A, V):=\{f: f \in C(X, K), f(A) \subset V+g\}$,

$B_{3}(g, A, V):=\{f: f \in C(X, K), f(A) \subset g V\}$,

$B_{4}(g, A, V):=\{f: f \in C(X, K), f(A) \subset V g\}$,

where $g \in C(X, K), A \in H, V \in \tau_{K}$. Evidently, the addition $+=\mu_{1}$ and the multiplication $\times=\mu_{2}$ are continuous relative to this topology, since each $U \in \tau_{C}$ is the union of base sets $P \in \beta_{C}$. In view of Section $5 C(X, K)$ is directed: $\mu_{p}(f, h) \leq \mu_{p}(g, u)$ for each $p=1,2$ and for every $f, g, h, u \in C(X, K)$ so that $f \leq g$ and $h \leq u$ when $f, g, h, u$ belong to the same linearly ordered set in $C(X, K)$, since element-wise these inequalities are satisfied in $K$, i.e. for $f(x), g(x), h(x), u(x)$ with $x \in X$ (see $\S 1$ ).

10. Note. Henceforward, it will be supposed that $C(X, K)$ is supplied with the topology $\tau_{C}$ of Lemma 9, while $C_{+}(X, K)$ and $C_{-}(X, K)$ are considered relative to the topology inherited from $C(X, K)$. Particularly, if $X \in H_{X}$, then it provides the topology of the uniform convergence on $C(X, K)$.

11. Corollary. If the conditions of Lemma 9 are satisfied and $H=2^{X}$ is the family of all subsets in $X$ and a topology $\tau_{K}$ on $K$ is discrete, then $\tau_{C}$ is the discrete topology on $C(X, K)$.

12. Lemma. Suppose that the conditions of Lemma 9 are satisfied. Then the functions

(1) $f \vee g(x):=\max (f(x), g(x))$ and

(2) $f \wedge g(x):=\min (f(x), g(x))$

are in $C(X, K)$ (or in $C_{+}(X, K)$ or in $C_{-}(X, K)$ ) for every pair of functions $f, g \in C(X, K)$ (or in $C_{+}(X, K)$ or in $C_{-}(X, K)$ correspondingly) satisfying the condition:

(3) for each $x \in X$ either $f(x)<g(x)$ or $g(x)<f(x)$ or $f(x)=g(x)$.

Proof. Let $f, g \in C(X, K)$ satisfy Condition (3). Then the sets $\{x: x \in X, f(x) \leq g(x)\}$ and $\{x: x \in X, f(x) \leq g(x)\}$ are closed in $X$, since $f$ and $g$ are continuous functions on $X$ and the topology $\tau_{K}$ on $K$ satisfies Condition 4(2). For each closed set $E$ in $K$ the sets

$(f \vee g)^{-1}(E)=\left[f^{-1}(E) \cap\{x: x \in X, g(x) \leq f(x)\}\right] \cup\left[g^{-1}(E) \cap\{x: x \in X, f(x) \leq g(x)\}\right]$ and

$(f \wedge g)^{-1}(E)=\left[f^{-1}(E) \cap\{x: x \in X, f(x) \leq g(x)\}\right] \cup\left[g^{-1}(E) \cap\{x: x \in X, g(x) \leq f(x)\}\right]$

are closed in $X$, consequently, the mappings $f \vee g$ and $f \wedge g$ are continuous. If $f, g \in C_{+}(X, K)$ and $x<y \in X$, then $f(x) \leq f(y)$ and $g(x) \leq g(y)$. If $f(x) \leq g(x)$ and $g(y) \leq f(y)$, then $(f \vee g)(x)=$ $g(x) \leq g(y) \leq f(y)=(f \vee g)(y)$ and $(f \wedge g)(x)=f(x) \leq g(x) \leq g(y)=(f \wedge g)(y)$. If $f(x) \leq g(x)$ and $f(y) \leq g(y)$, then $(f \vee g)(x)=g(x) \leq g(y)=(f \vee g)(y)$ and $(f \wedge g)(x)=f(x) \leq f(y)=$ $(f \wedge g)(y)$. Therefore, $(f \vee g)(x) \leq(f \vee g)(y)$ and $(f \wedge g)(x) \leq(f \wedge g)(y)$ for each $x<y \in X$. Thus $f \vee g$ and $f \wedge g \in C_{+}(X, K)$. Analogously if $f, g \in C_{-}(X, K)$, then $f \vee g$ and $f \wedge g \in C_{-}(X, K)$.

Relative to the topology of $\S 9$ on $C(X, K)$ operations $\vee$ and $\wedge$ are continuous on $C(X, K)$, $C_{+}(X, K)$ and $C_{-}(X, K)$.

\subsection{Examples of Directed Ringoids}

13. Example. Ringoids and ordinals. The class $O n$ of all ordinals has the addition $\mu_{1}=+_{o}$ and the multiplication $\mu_{2}=x_{0}$ operations which are generally non-commutative, associative, with unit elements 0 and 1 respectively, on $O n$ the right distributivity is satisfied (see Propositions 4.29-4.31 
and Examples $1-3$ in $[19,22])$. Relative to the interval topology generated by the base $\{(a, b)$ : $a<b \in O n\}$ the class $O n$ is the topological well-ordered semiring, where $(a, b)=\{c: c \in O n$, $a<c<b\}$. For each non-void set $A$ in $O n$ there exists sup $A \in O n$ (see [20]).

14. Example. Construction of ringoids with the help of inductive limits. Let $J$ be a directed set of the cardinality $\operatorname{card}(J) \geq \aleph_{0}$ such that for each $l, k \in J$ there exists $j \in J$ with $l \leq j$ and $k \leq j$ (see also §I.3 [21]), and let $\phi: J \rightarrow J$ be a monotone decreasing map, $G_{j} \subseteq[0, \infty)$, let also $p_{j}^{k}: G_{k} \rightarrow G_{j}$ be an embedding for each $k \leq j \in J$. There is considered $G_{j}$ as a ringoid with the addition, the multiplication, with neutral elements $0_{j}=0$ by addition and $1_{j}=1$ by multiplication and the linear ordering $x_{j}<y_{j}$ inherited from $[0, \infty)=\{t: t \in \mathbf{R}, 0 \leq t<\infty\}$ for each $j \in J$. Put $G_{0}=\lim \left\{G_{j}, p_{j}^{k}, J\right\}$ to be the inductive limit of the direct mapping system so that $G$ is the quotient $\left(\bigoplus_{j} G_{j}\right) / \Xi$ of the direct sum $\bigoplus_{j} G_{j}$ by the equivalence relation $\Xi$ caused by mappings $p_{j}^{k}$. Then consider $G:=\{x: x \in$ $\left.G_{0}, \sup _{j \in J} x_{j}<\infty\right\}$, where $x_{j}=p_{j}(x), p_{j}: G \rightarrow G_{j}$ notates the projection.

Then we define $g+{ }_{1} h:=\left\{v_{j}: v_{j}=g_{j}+h_{j} \forall j \in J\right\}$ and $g \times{ }_{1} h:=\left\{w_{j}: w_{j}=g_{j} p_{j}^{k}\left(h_{k}\right) \forall j \in\right.$ $J$ with $k=\phi(j)\}$ for all $g, h \in G$, where $g_{j}=p_{j}(g)$ for each $j \in J$. Let also $x<_{1} y$ in $G$ if and only if $x_{j}<y_{j}$ for each $j \in J$. Certainly for each $x, y \in G$ there exists $z \in G$ so that $x \leq_{1} z$ and $y \leq_{1} z$, for example, $z_{j}=\max \left(x_{j}, y_{j}\right)$ for each $j \in J$. Therefore we get that if $x<_{1} y$ and $u<_{1} z$ in $G$, then $x+{ }_{1} u<_{1} y+_{1} z$ and $x \times_{1} u<_{1} y \times_{1} z$. We supply $G$ with a topology $\tau_{b}$ inherited from the inductive limit topology on $G_{0}$, where $[0, \infty)$ is supplied with the standard metric of $\mathbf{R}$ and $G_{j}$ has the topology inherited from $[0, \infty)$. Then we deduce that $U\left(x_{j}, b, j\right)+U\left(z_{j}, b, j\right) \subset U\left(x_{j}+z_{j}, 2 b, j\right)$ and $U\left(x_{j}, b, j\right) U\left(z_{k}, b, k\right) \subset U\left(x_{j} p_{j}^{k}\left(z_{k}\right), b\left(1+x_{j}+z_{k}\right), j\right)$ for every $x, z \in G$ and $b>0$ and $j \in J$ with $k=\phi(j)$, where $U\left(x_{j}, b, j\right):=\left\{y_{j}: y_{j} \in G_{j}, x_{j}-b<y_{j}<x_{j}+b\right\}$. Since $\sup _{j \in J} x_{j}<\infty$ for each $x \in G$, then the addition and the multiplication in $G$ are continuous. Thus $\left(G,+_{1}, \times_{1},<_{1}, \tau_{b}\right)$ is the topological directed ringoid with the left and the right distributivity in which the multiplication $\times_{1}$ is non-associative, since $\phi(j)<j$ for each $j \in J$. It is worth to note that each set of the form $S(x):=\{y: y \in G$, either $x<y$ or $x$ is incomparable with $y\}$ is open in $\left(G, \tau_{b}\right)$, where $x \in G$.

15. Example. The case of $G_{j} \subseteq[0, \infty)^{\omega}$ for each $j \in J$, where $\omega$ is a directed set, can be considered analogously to Example 14, taking the lexicographic ordering on the Cartesian product $M:=\omega \times J$ and considering $M$ instead of $J$.

16. Example. On $G$ from Example 14 one can take also $x+2 y:=\left\{v_{j}: v_{j}=\max \left(x_{j}, y_{j}\right) \forall j \in J\right\}$ and $x \times_{2} y:=\left\{w_{j}: w_{j}=\min \left(x_{j}, y_{k}\right) \forall j \in J\right.$ with $\left.k=\phi(j)\right\}$. Then $\left(G,+_{2}, \times_{2},<_{1}, \tau_{b}\right)$ is a topological non-associative ringoid with the left and right distributivity.

17. Example. Ringoids associated with families of measures. Let $G_{j}$ be a Boolean algebra on a set $H_{j}$ and let $p_{j}^{k}: G_{k} \rightarrow G_{j}$ be an embedding for each $j \in J$ with $k=\phi(j)$ so that $m_{j}\left(p_{j}^{k}\left(C_{k}\right)\right) \leq m_{k}\left(C_{k}\right)$ for each $C_{k} \in G_{k}$, where $J$ and $\phi$ are as in subsection 14. Suppose that on each Boolean algebra $G_{j}$ there is a probability (finitely additive) measure $m_{j}: G_{j} \rightarrow[0,1]$ so that $G_{j}$ is metrizable by the metric $d_{j}\left(A_{j}, B_{j}\right):=m_{j}\left(A_{j} \triangle B_{j}\right)$, where $A_{j} \triangle B_{j}:=\left(A_{j} \backslash B_{j}\right) \cup\left(B_{j} \backslash A_{j}\right)$. Otherwise it is possible to consider the quotient algebra $G_{j} / \Xi_{j}$, where $A_{j} \Xi_{j} B_{j}$ if and only if $d_{j}\left(A_{j}, B_{j}\right)=0$. Put $A+B:=\{C$ : $\left.C_{j}=A_{j} \cup B_{j} \forall j \in J\right\}$ and $A \times B:=\left\{C: C_{j}=A_{j} \cap p_{j}^{k}\left(B_{k}\right) \forall j \in J\right.$ with $\left.k=\phi(j)\right\}$, where $A, B \in G$, $A_{j}, B_{j} \in G_{j}, G=\lim \left\{G_{j}, p_{j}^{k}, J\right\}$ is the inductive limit of Boolean algebras, $A_{j}=p_{j}(A), p_{j}: G \rightarrow G_{j}$ denotes the projection.

Consider on $G$ the inductive limit topology $\tau_{b}$, where $G_{j}$ is supplied with the metric $d_{j}$ for each $j \in J$. Naturally it is possible to put $A \leq B$ in $G$ if and only if $A_{j} \subseteq B_{j}$ for each $j \in J$. Then the inequalities

$$
\begin{aligned}
& \qquad m_{j}\left(\left(A_{j} \cup C_{j}\right) \triangle\left(B_{j} \cup D_{j}\right)\right) \leq m_{j}\left(\left(A_{j} \triangle B_{j}\right) \cup\left(C_{j} \triangle D_{j}\right)\right) \leq m_{j}\left(A_{j} \triangle B_{j}\right)+m_{j}\left(C_{j} \triangle D_{j}\right) \text { and } \\
& \quad m_{j}\left(\left(A_{j} \cap p_{j}^{k}\left(C_{k}\right)\right) \triangle\left(B_{j} \cap p_{j}^{k}\left(D_{k}\right)\right) \leq m_{j}\left(A_{j} \triangle B_{j}\right)+m_{j}\left(p_{j}^{k}\left(C_{k}\right) \triangle p_{j}^{k}\left(D_{k}\right)\right) \leq m_{j}\left(A_{j} \triangle B_{j}\right)+\right. \\
& m_{k}\left(C_{k} \triangle D_{k}\right) \\
& \text { are fulfilled for each } A_{j}, B_{j} \in G_{j} \text { and } C_{k}, D_{k} \in G_{k} \text {. Therefore }\left(G,+, \times,<, \tau_{b}\right) \text { is the topological ringoid } \\
& \text { with the left and right distributivity and the non-associative multiplication. }
\end{aligned}
$$


Instead of measures it is possible more generally to consider submeasures $m_{j}$, that is possessing the subadditivity property: $m_{j}\left(C_{j}\right) \leq m_{j}\left(A_{j}\right)+m_{j}\left(B_{j}\right)$ for each $A_{j}, B_{j}, C_{j} \in G_{j}$ satisfying the inclusion $C_{j} \subset A_{j} \cup B_{j}$.

18. Example. Ringoids induced by spectra of non locally compact groups. Let $\left\{G_{j}, p_{j}^{k}, J\right\}$ be a family of topological non locally compact groups $G_{j}$, where $J$ is a directed set, $p_{j}^{k}: G_{k} \rightarrow G_{j}$ is a continuous injective homomorphism for each $j<k$ in $J$. Let also $\phi: J \rightarrow J$ be an increasing map and let $m_{j}: \mathcal{B}_{j} \rightarrow[0,1]$ be a Radon probability $\sigma$-additive measure on the Borel $\sigma$-algebra $\mathcal{B}_{j}$ of $G_{j}$ such that $m_{j}$ is left quasi-invariant relative to $p_{j}^{k}\left(G_{k}\right)$ for each $j \in J$ with $k=\phi(j)$. That is there exists the Radon-Nikodym derivative (i.e., the left quasi-invariance factor) $d_{m}(v, g):=m^{v}(d g) / m(d g)$ for each $m=m_{j}$, where $v \in G_{k}, g \in G_{j}, m^{v}(A):=m\left(\left(p_{j}^{k}(v)\right)^{-1} A\right)$ for each $A \in A f\left(G_{j}, m_{j}\right)$, where $A f\left(G_{j}, m_{j}\right)$ denotes a $\sigma$-algebra which is the completion of $\mathcal{B}_{j}$ by $m_{j}$-null sets.

It is assumed that a uniformity $\tau_{G_{j}}$ on $G_{j}$ is such that $\tau_{G_{j}} \mid G_{k} \subset \tau_{G_{k}}$ and $\left(G_{j}, \tau_{G_{j}}\right)$ is complete for each $j \in J$ with $k=\phi(j)$. Suppose also that there exists an open base of neighborhoods of $e_{k} \in G_{k}$ such that their closures in $G_{j}$ are compact.

It is known that such systems exist for loop groups and groups of diffeomorphisms and Banach-Lie groups.

Then $L_{G_{k}}^{p}\left(G_{j}, m_{j}, \mathbf{R}\right)$ for $1 \leq p \leq \infty$ denotes the Banach space of all $m_{j}$-measurable functions $f: G_{j} \rightarrow \mathbf{R}$ such that $f^{h}(g) \in L^{p}\left(G_{j}, m_{j}, \mathbf{R}\right)$ for each $h \in G_{k}$ and

$$
\|f\|_{L_{G_{k}}^{p}\left(G_{j}, m_{j}, \mathbf{R}\right)}:=\sup _{h \in G_{k}}\left\|f^{h}\right\|_{L^{p}\left(G_{j}, m_{j}, \mathbf{R}\right)}<\infty
$$

where $f^{h}(g):=f\left(\left(p_{j}^{k}(h)\right)^{-1} g\right)$ for each $g \in G_{j}$ and $h \in G_{k}, j \in J$ with $k=\phi(j)$. Next we consider the space

$L^{\infty}\left(L_{G_{k}}^{1}\left(G_{j}, m_{j}, \mathbf{R}\right): j \in J, k=\phi(j)\right):=\left\{f=\left(f_{j}: j \in J\right) ; f_{j} \in L_{G_{k}}^{1}\left(G_{j}, m_{j}, \mathbf{R}\right)\right.$ for each $j \in J ;$ $\|f\|_{\infty}:=\sup _{j \in J}\left\|f_{j}\right\|_{L_{G_{k}}^{1}\left(G_{j}\right)}<\infty$, where $\left.k=\phi(j)\right\}$.

There exists the non-associative normed algebra $\mathcal{E}:=L^{\infty}\left(L_{G_{k}}^{1}\left(G_{j}, m_{j}, \mathbf{R}\right): j \in J, k=\phi(j)\right)$ supplied with the multiplication

$f \tilde{\star} u=w$ such that

$$
w_{j}\left(g_{j}\right)=\left(f_{k} \tilde{\star} u_{j}\right)\left(g_{j}\right)=\int_{G_{k}} f_{k}\left(t_{k}\right) u_{j}\left(p_{j}^{k}\left(t_{k}\right) g_{j}\right) m_{k}\left(d t_{k}\right)
$$

for every $f, u \in \mathcal{E}$ and $g \in G=\prod_{\alpha \in J} G_{\alpha}$, where $k=\phi(j), j \in J, g_{j} \in G_{j}$ (see [11-13,23]).

Now we take the positive cone

$F:=\left\{f: f \in \mathcal{E}, \forall j \in J f_{j}\left(g_{j}\right) \geq 0\right.$ for $m_{j}-$ almost all $\left.g_{j} \in G_{j}\right\}$ in $\mathcal{E}$ and put

$f+h=\left\{(f+h)_{j}\left(g_{j}\right)=f_{j}\left(g_{j}\right)+h_{j}\left(g_{j}\right) \forall j \in J \forall g_{j} \in G_{j}\right\}$,

$f \times h=f \tilde{\star} h$ for each $f, h \in F$ and define

$u \leq f$ in $F$ if and only if $u_{j}\left(g_{j}\right) \leq f_{j}\left(g_{j}\right)$ for each $j \in J$ and $m_{j}$-almost all $g_{j} \in G_{j}$. Therefore, $w_{j}\left(g_{j}\right) \geq 0$ for every $f, u \in F, j \in J$ and $m_{j}$-almost all $g_{j} \in G_{j}$, where $w=f \times u$, since $m_{k}$ is the probability measure, $f_{k}\left(t_{k}\right) \geq 0$ and $u_{j}\left(p_{j}^{k}\left(t_{k}\right) g_{j}\right) \geq 0$ for $m_{k}$-almost all $t_{k} \in G_{k}$ and $m_{j}$-almost all $g_{j} \in G_{j}$ correspondingly. Thus $f \times u \in F$ for each $f, u \in F$.

If $f, h, q, u \in F$ and $f \leq q, h \leq u$, then $f_{j}\left(g_{j}\right)+h_{j}\left(g_{j}\right) \leq q_{j}\left(g_{j}\right)+u_{j}\left(g_{j}\right)$ and

$$
\begin{aligned}
& \left(f_{k} \tilde{\star} h_{j}\right)\left(g_{j}\right)=\int_{G_{k}} f_{k}\left(t_{k}\right) h_{j}\left(p_{j}^{k}\left(t_{k}\right) g_{j}\right) m_{k}\left(d t_{k}\right) \\
\leq & \int_{G_{k}} q_{k}\left(t_{k}\right) u_{j}\left(p_{j}^{k}\left(t_{k}\right) g_{j}\right) m_{k}\left(d t_{k}\right)=\left(q_{k} \tilde{\star} u_{j}\right)\left(g_{j}\right)
\end{aligned}
$$


for $m_{j}$-almost all $g_{j} \in G_{j}$ and hence $f+h \leq q+u$ and $f \times h \leq q \times u$. Then we infer that

$$
\begin{gathered}
\left(\left(f_{k}+h_{k}\right) \tilde{\star} u_{j}\right)\left(g_{j}\right)=\int_{G_{k}}\left(f_{k}\left(t_{k}\right)+h_{k}\left(t_{k}\right)\right) u_{j}\left(p_{j}^{k}\left(t_{k}\right) g_{j}\right) m_{k}\left(d t_{k}\right) \\
=\int_{G_{k}} f_{k}\left(t_{k}\right) u_{j}\left(p_{j}^{k}\left(t_{k}\right) g_{j}\right) m_{k}\left(d t_{k}\right)+\int_{G_{k}} h_{k}\left(t_{k}\right) u_{j}\left(p_{j}^{k}\left(t_{k}\right) g_{j}\right) m_{k}\left(d t_{k}\right) \\
=\left(f_{k} \tilde{\star} u_{j}\right)\left(g_{j}\right)+\left(h_{k} \tilde{\star} u_{j}\right)\left(g_{j}\right)
\end{gathered}
$$

for every $f, h, u \in \mathcal{E}$ and $g \in G=\prod_{i \in J} G_{i}$, where $k=\phi(j), j \in J, \pi_{j}(g)=g_{j} \in G_{j}, \pi_{j}: G \rightarrow G_{j}$ is the projection, consequently, $(f+h) \times u=(f \times u)+(h \times u)$. Analogously it can be verified that $u \times(f+h)=(u \times f)+(u \times h)$ for every $f, h, u \in \mathcal{E}$.

For each $f, h \in F$ there exists an element $u \in F$ so that $f \leq u$ and $h \leq u$, for example, either $u=f+h$ or $u$ given by the formula $u_{j}\left(g_{j}\right)=\max \left(f_{j}\left(g_{j}\right), h_{j}\left(g_{j}\right)\right)$ for each $j \in J$ and $m_{j}$-almost all $g_{j} \in G_{j}$.

Take on $F$ the topology $\tau_{n}$ inherited from the norm topology on $\mathcal{E}$. This implies that $(F,+, \times,<$ ,$\left.\tau_{n}\right)$ is the directed topological non-associative ringoid with the left and right distributivity.

There is the decomposition $f=f^{+}-f^{-}$for each $f \in \mathcal{E}$, where $f^{+} \in F$ and $f^{-} \in F, f_{j}^{+}\left(g_{j}\right):=$ $\max \left(f_{j}\left(g_{j}\right), 0\right)$ for each $j \in J$ and $g_{j} \in G_{j}$.

If $f$ and $h$ in $F$ are incomparable, there exist $j, l \in J$ (may be either $j=l$ or $j \neq l$ ) such that $m_{j}\left(A_{j}^{+}\right)>0$ and $m_{l}\left(A_{l}^{-}\right)>0$, where

$A_{j}^{+}=A_{j}^{+}(f, h):=\left\{g_{j}: g_{j} \in G_{j}, f_{j}\left(g_{j}\right)>h_{j}\left(g_{j}\right)\right\}$ and

$A_{l}^{-}=A_{l}^{-}(f, h):=\left\{g_{l}: g_{l} \in G_{l}, f_{l}\left(g_{l}\right)<h_{l}\left(g_{l}\right)\right\}$. Then for

$0<b<\min \left(m_{j}\left(A_{j}^{+}\right), m_{l}\left(A_{l}^{-}\right)\right) \min \left(1,\left\|\left.\left(f_{j}-h_{j}\right)\right|_{A_{j}^{+}}\right\|_{L^{1}\left(A_{j}^{+}\right)},\left\|\left.\left(f_{l}-h_{l}\right)\right|_{A_{l}^{-}}\right\|_{L^{1}\left(A_{l}^{-}\right)}\right) / 4$

each element $v$ in the ball $B(F, h, b):=\left\{q: q \in F,\|q-h\|_{\mathcal{E}}<b\right\}$ is incomparable with $f$, since $\left\|q_{j}\right\|_{L^{1}\left(G_{j}\right)} \leq\left\|q_{j}\right\|_{L_{G_{k}}^{1}\left(G_{j}\right)}$ for each $q \in \mathcal{E}$ and $j \in J$, while $m_{j}$ is the probability measure for each $j \in J$.

On the other hand, if $v<u$ in $F$, there exists $l \in J$ so that $m_{l}\left(A_{l}^{-}(v, u)\right)>0$ and $v_{j}\left(g_{j}\right) \leq u_{j}\left(g_{j}\right)$ for $m_{j}$-almost all $g_{j} \in G_{j}$ for each $j \in J$. Therefore, for $0<b$ prescribed by the inequality given above and each $q \in B(F, h, b)$ the inequality $q \leq f$ is impossible, consequently, either $q$ is incomparable with $f$ or $f<q$. Thus each set of the form $S(f):=\{h: h \in F$, either $f<h$ or $f$ is incomparable with $h\}$ is open in $\left(F, \tau_{n}\right)$, where $f \in F$.

19. Note. Certainly relative to the discrete topology the aforementioned ringoids are also topological ringoids. Other examples can be constructed from these using the theorems and the propositions presented above.

\section{Skew Morphisms of Ordered Semirings and Ringoids}

\subsection{Morphisms and Their Properties}

1. Notation. Let $\times_{2}$ denote the mapping on $[K \times C(X, K)] \cup[C(X, K) \times K]$ with values in $C(X, K)$ such that

(1) $c \times{ }_{2} f:=g^{c}+f=: g^{c} \times_{2} f$ and $f \times{ }_{2} c:=f+g^{c}=: f \times_{2} g^{c}$ for each $c \in K$ and $f \in C(X, K)$, where $g^{c}(x):=c$ for each $x \in X$, whilst the sum is taken element-wise $(f+g)(x)=f(x)+g(x)$ for every $f, g \in C(X, K)$ and $x \in X$.

2. Definition. We call a mapping $v$ on $C(X, K)$ (or $C_{+}(X, K)$ or $C_{-}(X, K)$ ) with values in $K$ an idempotent (K-valued) morphism if it satisfies for each $f, g, g^{c} \in C(X, K)$ (or in $C_{+}(X, K)$ or $C_{-}(X, K)$ correspondingly) the following five conditions

(1) $v\left(g^{c}\right)=c$;

(2) $v\left(c \times_{2} f\right)=c+v(f)=: c \times_{2} v(f)$ and

(3) $v\left(f \times \times_{2} c\right)=v(f)+c=: v(f) \times{ }_{2} c$;

(4) $v(f \vee g)=v(f) \vee v(g)$ when $f, g$ satisfy Condition 2.12(3) and 
(5) $v(f \wedge g)=v(f) \wedge v(g)$ if $f, g$ satisfy Condition 2.12(3),

where $a \vee b=\max (a, b)$ and $a \wedge b=\min (a, b)$ for each $a, b \in K$ when either $a<b$ or $a=b$ or $b<a$.

A mapping (morphism) $v$ on $C(X, K)$ (or $C_{+}(X, K)$ or $C_{-}(X, K)$ ) with values in $K$ we call order preserving (non-decreasing), if

(6) $v(f) \leq v(g)$ for each $f \leq g$

in $C(X, K)$ (or $C_{+}(X, K)$ or $C_{-}(X, K)$ respectively), i.e., when $f(x) \leq g(x)$ for each $x \in X$.

A morphism $v$ will be called $K$-homogeneous on $C(X, K)\left(\right.$ or $C_{+}(X, K)$ or $\left.C_{-}(X, K)\right)$ if

(7) $v(b f)=b v(f)$ and

(8) $v(f b)=v(f) b$

for each $f$ in $C(X, K)$ (or $C_{+}(X, K)$ or $C_{-}(X, K)$ correspondingly) and $b \in K$.

3. Remark. If a morphism satisfies Condition 2(4), then it is order preserving.

The evaluation at a point morphism $\delta_{x}$ defined by the formula:

(1) $\delta_{x} f=f(x)$

is the idempotent $K$-homogeneous morphism on $C(X, K)$, where $x$ is a marked point in $X$.

If morphisms $v_{1}, \ldots, v_{n}$ are idempotent and the multiplication in $K$ is distributive, then for each constants

(2) $c_{1}>0, \ldots, c_{n}>0$ in $K$ with

(3) $c_{1}+\ldots+c_{n}=1$ morphisms

(4) $c_{1} v_{1}+\ldots+c_{n} v_{n}$ and

(5) $v_{1} c_{1}+\ldots+v_{n} c_{n}$

are idempotent. Moreover, if the multiplication in $K$ is commutative, associative and distributive and constants satisfy Conditions $(2,3)$ and morphisms $v_{1}, \ldots, v_{n}$ are $K$-homogeneous, then morphisms of the form $(4,5)$ are also $K$-homogeneous.

The considered here theory is different from the usual real field $\mathbf{R}$, since $\mathbf{R}$ has neither an infimum nor a supremum, i.e. it is not well-ordered and satisfy neither 2.3(DW) nor 2.4(1).

4. Lemma. Suppose that either

(1) $K$ is well-ordered and satisfies Conditions $2.4(1-3)$ or

(2) $X$ is linearly ordered and $K$ is directed and satisfies Conditions $2.3(D W)$ and $2.4(1-3)$. Then there exists an idempotent $K$-homogeneous morphism $v$ on $C(X, K)$ in case $(1)$, on $C_{+}(X, K)$ and $C_{-}(X, K)$ in case (2). Moreover, if $K \subset$ On and $K$ is infinite, $X$ is not a singleton, $\aleph_{0} \leq|K|,|X|>1$, then $v$ has not the form either 3(4) or 3(5) with the evaluation at a point morphisms $v_{1}, \ldots, v_{n}$ relative to the standard addition in On.

Proof. Suppose that $v$ is an order preserving morphism on $C(X, K)\left(\right.$ or $C_{+}(X, K)$ or $\left.C_{-}(X, K)\right)$. If $f, g$ in $C(X, K)$ (or $C_{+}(X, K)$ or $C_{-}(X, K)$ respectively) satisfy Condition 2.12(3), then in accordance with Lemma 2.12 there exists $f \vee g$ and $f \wedge g$ in the corresponding $C(X, K)$ (or $C_{+}(X, K)$ or $C_{-}(X, K)$ ). Since $f \vee g \geq f$ and $f \vee g \geq g$ and $f \wedge g \leq f$ and $f \wedge g \leq g$ and the morphism $v$ is order preserving, then $v(f) \vee v(g) \leq v(f \vee g)$ and $v(f \wedge g) \leq v(f) \wedge v(g)$.

Let also $E$ be a subset in $X$, we put

(3) $v(f)=v_{E}(f)=\sup _{x \in E} f(x)$.

This morphism exists due Conditions 2.4(1,3), since in both cases (1) and (2) of this lemma, the image $f(E)$ is linearly ordered and is contained in $K$.

From the fact that the addition preserves ordering on $K$ (see \$2.1) it follows that Properties $(1-3,7,8)$ are satisfied for the morphism $v$ given by Formula (3). If $f \leq g$ on $X$, then for each $a \in f(E)$ there exists $b \in g(E)$ so that $a \leq b$, consequently, $v(f) \leq v(g)$, i.e., $2(6)$ is fulfilled.

We consider any pair of functions $f, g$ in $C(X, K)$ (or $C_{+}(X, K)$ or $C_{-}(X, K)$ ) satisfying Condition 8 (3). In case (2) a topological space $X$ is linearly ordered, in case (1) $K$ is well-ordered, hence $f(X)$, $g(X), f(E)$ and $g(E)$ are linearly ordered in $K$. Then for each $a \in f(E) \cup g(E)$ there exist $b \in(f \vee g)(E)$ so that $a \leq b$, while for each $c \in(f \vee g)(E)$ there exists $d \in f(E) \cup g(E)$ so that $c \leq d$, hence $v(f \vee g)=$ $v(f) \vee v(g)$. Moreover, for each $a \in f(E) \cup g(E)$ there exists $b \in(f \wedge g)(E)$ so that $b \leq a$ and for each 
$c \in(f \wedge g)(E)$ there exists $d \in f(E) \cup g(E)$ so that $d \leq c$, consequently, $v(f \wedge g)=v(f) \wedge v(g)$. Thus Properties $2(4,5)$ are satisfied as well.

If $E$ is chosen such that there exists $U \in H_{X}$ with $E \subset U$, then this morphism $v$ is continuous on $C(X, K), C_{+}(X, K)$ and $C_{-}(X, K)$ (see $\S \S 2.3,2.4,2.9$ and 2.10 also).

If a set $X$ is not a singleton, $|X|>1$, and $K \subset O n$ is infinite, $\aleph_{0} \leq|K|$, then taking a set $E$ in $X$ different from a singleton, $|E|>1$, we get that the morphism given by Formula (3) can not be presented with the help of evaluation at a point morphisms $v_{1}=\delta_{x_{1}}, \ldots, v_{n}=\delta_{x_{n}}$ by Formula either $3(4)$ or 3(5) relative to the standard addition in $O n$, since functions $f$ in $C(X, K)$ (or $C_{+}(X, K)$ or $C_{-}(X, K)$ ) separate points in $X$ (see Remark 2.5(3)).

5. Remark. Relative to the idempotent addition $x \vee y=\max (x, y)$ the morphism $v_{E}$ given by 4(3) has the form $v_{E}(f)=\vee_{x \in E} \delta_{x}(f)$.

Let $I(X, K)$ denote the set of all idempotent $K$-valued morphisms, while $I_{h}(X, K)$ denotes its subset of idempotent homogeneous morphisms.

A set $F$ of all continuous $K$-valued morphisms on $C(X, K)$ is supplied with the weak topology having the base consisting of the sets

(1) $<\mu ; g_{1}, \ldots, g_{n} ; b>_{1}:=\left\{v: v \in F, \forall j=1, \ldots, n v\left(g_{j}\right)<\mu\left(g_{j}\right)+b\right\}$;

$<\mu ; g_{1}, \ldots, g_{n} ; b>_{2}:=\left\{v: v \in F, \forall j=1, \ldots, n \mu\left(g_{j}\right)<v\left(g_{j}\right)+b\right\} ;$

$<\mu ; g_{1}, \ldots, g_{n} ; b>_{3}:=\left\{v: v \in F, \forall j=1, \ldots, n v\left(g_{j}\right)<b+\mu\left(g_{j}\right)\right\} ;$

$<\mu ; g_{1}, \ldots, g_{n} ; b>_{4}:=\left\{v: v \in F, \forall j=1, \ldots, n \mu\left(g_{j}\right)<b+v\left(g_{j}\right)\right\}$

and their finite intersections, where $0<b \in K, g_{1}, \ldots, g_{n} \in C(X, K), \mu \in F$.

6. Definitions. A morphism $v: C(X, K) \rightarrow K$ is called weakly additive, if it satisfies Conditions 2(2,3);

normalized at $c \in K$, if Formula 2(1) is fulfilled;

(1) non-expanding if $v(f) \leq v(h)+c$ when $f \leq h+g^{c}$ and $v(f) \leq c+v(h)$ when $f \leq g^{c}+h$ for any $f, h \in C(X, K)$ and $c \in K$,

where $v$ may be non-linear or discontinuous as well.

The family of all order preserving weakly additive morphisms on a Hausdorff topological space $X$ with values in $K$ will be denoted by $\mathcal{O}(X, K)$.

If $E \subset C(X, K)$ satisfies the conditions: $g^{0} \in E, g+b$ and $b+g \in E$ for each $g \in E$ and $b \in K$, then $E$ is called an $A$-subset.

7. Lemma. If $v: C(X, K) \rightarrow K$ is an order preserving weakly additive morphism, then it is non-expanding.

Proof. Suppose that $f, h \in C(X, K)$ and $b \in K$ are such that $f(x) \leq(h(x)+c)$ or $f(x) \leq$ $(c+h(x))$ for each $x \in X$, then $2(2,3,6)$ imply that $v(f) \leq(v(h)+c)$ or $v(f) \leq(c+v(h))$ respectively. Thus the morphism $v$ is non-expanding.

8. Corollary. Suppose that a topological ringoid $K$ is well-ordered, satisfies 1(1) and with the interval topology, $X \in H, C(X, K)$ is supplied with the topology of $\$ 2.9$. Then any order preserving weakly additive morphism $v: C(X, K) \rightarrow K$ is continuous.

Proof. This follows from Lemma 7 and $\S \S 2.3,2.4$, since each subset $\{f: f \leq g\}$ and $\{f: g \leq f\}$ is closed in $C(X, K)$ in the topology of $\$ 2.9$, where $g \in C(X, K)$.

9. Lemma. Suppose that $A$ is an $A$-subset (a left or right submodule over $K$ ) in $C(X, K)$ and $v: A \rightarrow K$ is an order preserving weakly additive morphism (left or right $K$-homogeneous with left or right distributive ringoid $K$ correspondingly). Then there exists an order preserving weakly additive morphism $\mu: C(X, K) \rightarrow K$ such that its restriction on A coincides with $v$.

Proof. One can consider the set $\mathcal{F}$ of all pairs $(B, \mu)$ so that $B$ is an $A$-subset (a left or right submodule over $K$ respectively), $A \subseteq B \subseteq C(X, K), \mu$ is an order preserving weakly additive morphism on $B$ the restriction of which on $A$ coincides with $v$. The set $\mathcal{F}$ is partially ordered: $\left(B_{1}, \mu_{1}\right) \leq\left(B_{2}, \mu_{2}\right)$ if $B_{1} \subseteq B_{2}$ and $\mu_{2}$ is an extension of $\mu_{1}$. In accordance with Zorn's lemma a maximal element $(E, \mu)$ in $\mathcal{F}$ exists. 
If $E \neq C(X, K)$, there exists $g \in C(X, K) \backslash E$. Let $E_{-}:=\{f: f \in E, f \leq g\}$ and $E_{+}:=$ $\{f: f \in E, g \leq f\}$, then $\mu(h) \leq \mu(q)$ for each $h \in E_{-}$and $q \in E_{+}$, consequently, an element $b \in K$ exists such that $\mu\left(E_{-}\right) \leq b \leq \mu\left(E_{+}\right)$due to Conditions 2.3(DW) and 2.4(1) imposed on $K$. Then we put $F=E \cup\left\{g+g^{c}, g^{c}+g: c \in K\right\}$ ( $F$ is a minimal left or right module over $K$ containing $E$ and $g$ correspondingly). Then one can put $\mu\left(g+g^{c}\right)=b+c$ and $\mu\left(g^{c}+g\right)=c+b$. Moreover, one gets $\mu\left(d\left(g+g^{c}\right)\right)=d \mu(g)+d c$ or $\mu\left(\left(g+g^{c}\right) d\right)=\mu(g) d+c d$ for each $d \in K$ correspondingly for each $c \in K$. Then $\mu$ is an order preserving weakly additive morphism (left or right homogeneous correspondingly) on $F$. This contradicts the maximality of $A$.

10. Theorem. If a ringoid $K$ is well-ordered and satisfies $1(1)$, with the interval topology and $K$ is locally compact, $X \in H_{X}$. Then $\mathcal{O}(X, K)$ is compact relative to the weak* topology.

Proof. In view of Lemma 8 each $v \in \mathcal{O}(X, K)$ is continuous. The set $\mathcal{O}(X, K)$ is supplied with the weak* topology (see §5).

For each $v \in \mathcal{O}(X, K)$ one has $v\left(g^{c}\right)=v\left(g^{c}+g^{0}\right)=c$, since $g^{c}+g^{0}=g^{c}$ and $v\left(g^{c}+g^{0}\right)=$ $c+0$. On the other hand, for each $g \in C(X, K)$ due to Condition 2.4(1) a supremum exists, $\|g\|:=$ $\sup _{x \in X} g(x) \in K$. Each segment $[a, b]$ in $K$ is closed, bounded and hence compact relative to the interval topology. Therefore, $\mathcal{O}(X, K)$ is contained in the Tychonoff product $S=\prod\{[0,\|g\|]: g \in$ $C(X, K)\}$, since $g \leq h$ and hence $v(g) \leq v(h)$ when $h(x)=\|g\|$ for each $x \in X$. This product is compact as the Tychonoff product of compact topological spaces by Theorem 3.2.13 [21]. It remains to prove, that $\mathcal{O}(X, K)$ is closed in $S$, since a closed subspace of a compact topological space is compact (see Theorem 3.1.2 [21]).

Each compact Hausdorff space has a uniformity compatible with its topology (see Theorems 3.19 and 8.1.20 [21]). To each element $y \in S$ a morphism $y: C(X, K) \rightarrow K$ corresponds, since $[0,\|g\|] \subset K$ for each $g \in C(X, K)$. If $v_{n} \in \mathcal{O}(X, K)$ is a net converging to $q$ in $S$, then Properties $2(2,3,6)$ for each $v_{n}$ imply Properties $2(2,3,6)$ for $q$, since each segment $[a, b]$ in $K$ is compact and hence complete as the uniform space due to Theorem 8.3.15 [21], where $a<b \in K$. Therefore, $\lim _{n}=q \in \mathcal{O}(X, K)$ according to Lemma 7 and Corollary 8 . Thus $\mathcal{O}(X, K)$ is complete as the uniform space by Theorem 8.3.20 [21] and hence closed in $S$ in accordance with Theorem 8.3.6 [21].

11. Proposition. In the topological space $\mathcal{O}(X, K)$ the subsets $I(X, K)$ and $I_{h}(X, K)$ are closed.

Proof. From the definitions above it follows that $I_{h}(X, K) \subset I(X, K) \subset \mathcal{O}(X, K)$. If $v_{k}$ is a net in $I(X, K)$ (or in $I_{h}(X, K)$ ) converging to a morphism $\mu \in \mathcal{O}(X, K)$ relative to the weak topology (see also $\S 1.6[21]$ ), then $\mu$ satisfies Conditions $2(1-5)$ (or to $2(1-5,7,8)$ respectively). Thus $I(X, K)$ and $I_{h}(X, K)$ are closed in $\mathcal{O}(X, K)$.

12. Corollary. If the conditions of Theorem 10 are satisfied, then the topological spaces $I(X, K)$ and $I_{h}(X, K)$ are compact.

\subsection{Categories of Semirings, Ringoids and Morphisms}

13. Definition. If topological spaces $X$ and $Y$ are given and $f: X \rightarrow Y$ is a continuous mapping, then it induces the mapping $\mathcal{O}(f): \mathcal{O}(X, K) \rightarrow \mathcal{O}(Y, K)$ according to the formula: $(\mathcal{O}(f)(v))(g)=$ $v(g(f))$ for each $g \in C(Y, K)$ and $v \in \mathcal{O}(X, K)$.

By $I(f)$ will be denoted the restriction of $\mathcal{O}(f)$ onto $I(X, K)$.

A $T_{1}$ topological space will be called $K$-completely regular (or $K$ Tychonoff space), if for each closed subset $F$ in $X$ and each point $x \in X \backslash F$ a continuous function $h: X \rightarrow K$ exists such that $h(x)=0$ and $h(F)=\{c\}$, i.e. $h$ is constant on $F$, where $c \neq 0$.

Let $R K$ denote a category such that a family $O b(R K)$ of its objects consists of all $K$-regular topological spaces, a set of morphisms $\operatorname{Mor}(X, Y)$ consists of all continuous mappings $f: X \rightarrow Y$ for every $X, Y \in O b(R K)$, i.e. $R K$ is a subcategory in the category of topological spaces. We denote by $\mathcal{O} K$ a category with objects $O b(\mathcal{O} K)=\{\mathcal{O}(X, K): X \in O b(R K)\}$ and families of morphisms $\operatorname{Mor}(\mathcal{O}(X, K), \mathcal{O}(Y, K))$. 
14. Lemma. (1). There exists a covariant functor $\mathcal{O}$ in the category $R K$. (2). Moreover, if a topological ringoid $K$ is well-ordered, satisfies 2.4(1) and with the interval topology, when $f \in \operatorname{Mor}(X, Y), X \in H_{X}$, $Y \in H_{Y}, X, Y \in O b(R K)$, then $\mathcal{O}(f)$ is continuous.

Proof. (1). If $X, Y \in O b(R K)$ and $f \in \operatorname{Mor}(X, Y), g \leq h$ in $C(Y, K)$, then $g \circ f \leq h \circ f$ in $C(X, K)$, consequently, $(\mathcal{O}(f)(v))(g)=v(g \circ f) \leq v(h \circ f)=(\mathcal{O}(f)(v))(h)$ for each $v \in \mathcal{O}(X, K)$. If $c \in K$, $g^{c} \in C(Y, K)$, then $g^{c} \circ f \in C(X, K),(\mathcal{O}(f)(v))\left(g^{c}+h\right)=v\left(g^{c} \circ f+h \circ f\right)=c+v(h \circ f)=c+$ $(\mathcal{O}(f)(v))(h)$ and $(\mathcal{O}(f)(v))\left(h+g^{c}\right)=v\left(h \circ f+g^{c} \circ f\right)=v(h \circ f)+c=(\mathcal{O}(f)(v))(h)+c$ for each $h \in C(Y, K)$. If $1_{X} \in \operatorname{Mor}(X, X), 1_{X}(x)=x$ for each $x \in X$, then $1_{X} \circ q=q$ for each $q \in \operatorname{Mor}(Y, X)$ and $t \circ 1_{X}=t$ for each $t \in \operatorname{Mor}(X, Y)$. On the other hand, $\left(\mathcal{O}\left(1_{X}\right)(v)\right)(g)=v\left(g \circ 1_{X}\right)=v(g)$ for each $g \in C(X, K)$, i.e., $\mathcal{O}\left(1_{X}\right)=1_{\mathcal{O}(X)}$. Evidently, $(\mathcal{O}(f \circ s)(v))(g)=v(g \circ f \circ s)=(\mathcal{O}(s)(v)(g \circ f)=$ $((\mathcal{O}(f) \circ \mathcal{O}(s))(v))(g)$.

(2). If $v_{j}$ is a net converging to $v$ in $\mathcal{O}(X, K)$ relative to the weak topology, then $\lim _{j}\left(\mathcal{O}(f)\left(v_{j}\right)\right)(g)=\lim _{j} v_{j}(g \circ f)=v(g \circ f)=(\mathcal{O}(f)(v))(g)$ for each $f \in \operatorname{Mor}(X, Y)$ and $g \in C(Y, K)$, since $\mathcal{O}(X, K)$ and $\mathcal{O}(Y, K)$ are weakly* compact according to Theorem 10 , consequently, $\mathcal{O}$ is continuous from $\mathcal{O}(X, K)$ to $\mathcal{O}(Y, K)$.

15. Proposition. If $f \in \operatorname{Mor}(X, Y)$ for $X, Y \in O b(R K)$, then

$\mathcal{O}(f)(I(X, K)) \subseteq I(Y, K)$.

Proof. If $g, h \in C(Y, K)$ are such that $g \vee h$ or $g \wedge h$ exists and $f: X \rightarrow Y$ is a continuous mapping, then

$(\mathcal{O}(f)(v))(g \vee h)=v(g \circ f \vee h \circ f)=v(g \circ f) \vee v(h \circ f)=(\mathcal{O}(f)(v))(g) \vee(\mathcal{O}(f)(v))(h)$ or

$(\mathcal{O}(f)(v))(g \wedge h)=v(g \circ f \wedge h \circ f)=v(g \circ f) \wedge v(h \circ f)=(\mathcal{O}(f)(v))(g) \wedge(\mathcal{O}(f)(v))(h)$.

Then for each element $c \in K$ one gets

$(\mathcal{O}(f)(v))\left(g^{\mathcal{c}} \times{ }_{2} h\right)=v\left(g^{\mathcal{c}} \circ f \times_{2} h \circ f\right)=v\left(g^{\mathcal{c}} \circ f\right) \times_{2} v(h \circ f)=c \times_{2}(\mathcal{O}(f)(v))(h)$ and

$(\mathcal{O}(f)(v))\left(h \times{ }_{2} g^{c}\right)=v\left(h \circ f \times_{2} g^{c} \circ f\right)=v(h \circ f) \times_{2} v\left(g^{c} \circ f\right)=(\mathcal{O}(f)(v))(h) \times_{2} c$.

16. Definitions. A covariant functor $F: R K \rightarrow R K$ will be called epimorphic (monomorphic) if it preserves epimorphisms (monomorphisms). If $\phi: A \hookrightarrow X$ is an embedding, then $F(A)$ will be identified with $F(\phi)(F(A))$.

If for each $f \in \operatorname{Mor}(X, Y)$ and each closed subset $A$ in $Y$, the equality $\left(F(f)^{-1}\right)(F(A))=$ $F\left(f^{-1}(A)\right)$ is satisfied, then a covariant functor $F$ is called preimage-preserving. When $F\left(\bigcap_{j \in J} X_{j}\right)=$ $\bigcap_{j \in J} F\left(X_{j}\right)$ for each family $\left\{X_{j}: j \in J\right\}$ of closed subsets in $X \in O b(R K)$ the monomorphic functor $F$ is called intersection-preserving.

If a functor $F$ preserves inverse mapping system limits, it is called continuous.

A functor $F$ is said to be weight-preserving when $w(X)=w(F(X))$ for each $X \in O b(R K)$, where $w(X)$ denotes the topological weight of $X \in O b(R K)$.

A functor is said to be semi-normal when it is continuous, monomorphic, epimorphic, preserves weights, intersections, preimages and the empty space.

If a functor is continuous, monomorphic, epimorphic, preserves weights, intersections and the empty space, then it is called weakly semi-normal.

17. Lemma. Let $Y$ be a normal topological space, let also $A$ and $B$ be nonintersecting closed subsets in $Y$, where $T$ is a well-ordered set supplied with the interval topology. Suppose also that $c_{1}<c_{2} \in T$ are such that for each $a, b \in T$ with $c_{1} \leq a<b \leq c_{2}$ an element $d \in T$ exists such that $a<d<b$ (i.e. a segment $\left[c_{1}, c_{2}\right]$ is without gaps). Then a continuous function $f: Y \rightarrow$ T exists such that $f(A)=\left\{c_{1}\right\}$ and $f(B)=\left\{c_{2}\right\}$.

Proof. Consider the segment $\left[c_{1}, c_{2}\right]$ in $T$. There exists a set $E$ dense in $\left[c_{1}, c_{2}\right]$ such that

(1) $|E|=d\left(\left[c_{1}, c_{2}\right]\right), \inf E=c_{1}, \sup E=c_{2}$,

where $d(X)$ denotes the density of a topological space $X,|E|$ denotes the cardinality of $E$. There exist open subsets $U$ and $V$ in $X$ such that

(2) $A \subset U, B \subset V, U \cap V=\varnothing$.

We define open subsets $V_{t}$ in $X$ such that

(3) $c l_{X} V_{t} \subset V_{s}$ for each $t<s \in E$,

(4) $A \subset V_{c_{1}}, B \subset X \backslash V_{c_{2}}$, 
where $c l_{X} G$ denotes the closure of a set $G$ in $X$.

Sets $V_{t}$ will be defined by the transfinite induction. For this one can put $V_{c_{1}}=U$ and $V_{c_{2}}=X \backslash B$. Therefore, $A \subset V_{c_{1}} \subset X \backslash V=c l_{X}(X \backslash V) \subset V_{c_{2}}$, consequently, $c l_{X} V_{c_{1}} \subset V_{c_{2}}$. In view of the Zermelo theorem there exists an ordinal $P$ such that $|P|=|E|$, a bijective surjective mapping $\theta: P \rightarrow E$ exists such that $\inf P=0,1 \in P, \theta(0)=c_{1}$ and $\theta(1)=c_{2}$. Suppose that $V_{t_{j}}$ satisfying Condition (3) are constructed for $j=1, \ldots, n, j \in P$. There exist elements $a_{n}=\inf \left\{t_{j}: j \leq n, t_{j}<t_{n+1}\right\}$ and $b_{n}=\sup \left\{t_{j}: j \leq n, t_{j}<t_{n+1}\right\}$. Therefore, $c l_{X} V_{a_{n}} \subset V_{b_{n}}$. From the normality of $X$ it follows that open sets $U$ and $V$ exist such that $c l_{X} V_{a_{n}} \subset U, X \backslash V_{b_{n}} \subset V$ and $U \cap V=\varnothing$, consequently, $U \subset X \backslash V \subset V_{b_{n}}$ and hence $c l_{X} U \subset c l_{X}(X \backslash V)=X \backslash V \subset V_{b_{n}}$. Then one puts $V_{t_{n+1}}=U$. This means that there exists a countable infinite sequence $V_{t_{j}}$ for $j \in \omega_{0} \subseteq P$ satisfying Conditions $(3,4)$. If $\left\{t_{j}: j \in \omega_{0}\right\}$ is not dense in $\left[c_{1}, c_{2}\right]$ the process continues. Suppose that $\alpha$ is an ordinal such that $\omega_{0} \subseteq \alpha \subset P, V_{t_{j}}$ is defined for each $j \in \alpha$. If the set $\left\{t_{j}: j \in \alpha\right\}$ is not dense in $\left[c_{1}, c_{2}\right]$, there exists a segment

(5) $[a, b] \subset\left[c_{1}, c_{2}\right]$ such that $[a, b] \cap\left\{t_{j}: j \in \alpha\right\}=\varnothing$. We put $L=\bigcup_{t_{j}<a ; j \in \alpha} V_{t_{j}}$ and $M=\bigcap_{b<t_{j} ; j \in \alpha} V_{t_{j}}$. From $(3,4)$ it follows that the set $L$ is open in $X$ and $L \subset M$. On the other hand,

(6) $V_{t_{l}} \subset L \subset c l_{X} L \subset c l_{X} M \subset c l_{X} V_{t_{j}} \subset V_{t_{k}}$ for every $l, j, k \in \alpha$ such that $t_{l}<a$ and $b<t_{j}<t_{k}$. If

(7) $c l_{X} L$ is not contained in $\operatorname{Int}_{X} M$ this segment $[a, b]$ is skipped, where $\operatorname{Int}_{X} M$ is an interior of $M$ in $X$. If $c l_{X} L \subset \operatorname{Int}_{X} M$ one can put $V_{a}=L$ and $V_{b}=\operatorname{Int}_{X} M$. Then the process continues for $[a, b]$.

The family $\mathcal{F}=\left\{\left(V_{j}: j \in \alpha\right): \alpha \subset P\right\}$ is ordered by inclusion: $\left(V_{j}: j \in \alpha\right) \leq\left(W_{k}: k \in \beta\right)$ if and only if a bijective monotonously increasing mapping $\theta: \alpha \rightarrow \beta$ exists such that $V_{j}=W_{\theta(j)}$ for each $j \in \alpha$. If a subfamily $\left\{\left(V_{j}: j \in \alpha\right): \alpha_{k} \subset P, k \in \Lambda\right\}$ is linearly ordered, then its union is in $\mathcal{F}$. In view of the Kuratowski-Zorn lemma there exists a maximal element $\left(V_{j}: j \in \alpha_{1}\right)$ in $\mathcal{F}$ for some ordinal $\alpha_{1} \subset P$ such that conditions $(3,4)$ are satisfied.

Put $f(x)=\inf \left\{t: x \in V_{t}\right\}$ for $x \in V_{c_{2}}$ and $f(x)=c_{2}$ when $x \in X \backslash V_{c_{2}}$. Therefore, $f(x) \in\left[c_{1}, c_{2}\right]$ for each $x \in X, f(A) \subset\left\{c_{1}\right\}$ and $f(B) \subset\left\{c_{2}\right\}$. Since $\left[c_{1}, c_{2}\right]$ is supplied with the interval topology it is sufficient to prove that $f^{-1}\left(\left[c_{1}, a\right)\right)$ and $f^{-1}\left(\left(b, c_{2}\right]\right)$ are open in $\left[c_{1}, c_{2}\right]$ for each $c_{1}<a \leq c_{2}$ and $c_{1} \leq b<c_{2}$. From $(3,4)$, also from $(6,7)$ when (5) is fulfilled, and the definition of $f$ it follows that $f^{-1}\left(\left[c_{1}, a\right)\right)=\bigcup\left\{V_{t_{j}}: t_{j}<a, j \in \alpha_{1}\right\}$ and $f^{-1}\left(\left(b, c_{2}\right]\right)=\bigcup\left\{X \backslash c l_{X} V_{t_{j}}: b<t_{j}, j \in \alpha_{1}\right\}$ are open in $\left[c_{1}, c_{2}\right]$.

18. Lemma. If $X$ is well-ordered and $E$ is a segment $[a, b]$ in $X$, while $K$ satisfies Condition $2.3(D W)$, then each $f \in C_{+}(E, K)$ has a continuous extension $g \in C_{+}(X, \mathbf{K})$.

Proof. Since $f(E)=: A$ is linearly ordered in $K$, then by 2.3(DW) there exists a well ordered subset $B$ in $K$ such that $A \subset B$. So putting $g(x)=\inf A$ for each $x<a$ in $X$, whilst $g(x)=\sup A$ for each $b<x$ in $X$ one gets the continuous extension $g \in C_{+}(X, K)$ of $f$, that is $\left.g\right|_{E}(y)=f(y)$ for each $y \in E$, since $\inf A$ and $\sup A$ exist in $K$ due to $2.3(D W)$ and 2.4(1).

19. Definition. It will be said that a pair $(X, K)$ of a topological space $X$ and a ringoid $K$ has property $(C E)$ if for each closed subset $E$ in $X$ and each continuous function $f: E \rightarrow K$, i.e., $f \in C(E, K)$ or $f \in C_{+}(E, K)$ or $f \in C_{-}(E, K)$, there exists a continuous extension $g: X \rightarrow K$, i.e., $\left.g\right|_{E}=f$ so that $g \in C(X, K)$ or $g \in C_{+}(X, K)$ or $g \in C_{-}(X, K)$ respectively.

Henceforward, it will be supposed that a pair $(X, K)$ has property $(C E)$.

20. Definitions. If Hausdorff topological spaces $X$ and $Y$ are given and $f: X \rightarrow Y$ is a continuous mapping, $K_{1}, K_{2}$ are ordered topological ringoids (or may be particularly semirings) with an order-preserving continuous algebraic homomorphism $u: K_{1} \rightarrow K_{2}$ then it induces the mapping $\mathcal{O}(f, u): \mathcal{O}\left(X, K_{1}\right) \rightarrow \mathcal{O}\left(Y, K_{2}\right)$ according to the formula:

(1) $(\mathcal{O}(f, u)(v))(g)=u\left[v\left(g_{1}(f)\right)\right]$ for each $g_{1} \in C\left(Y, K_{1}\right)$ and $v \in \mathcal{O}\left(X, K_{1}\right)$, where $u \circ g_{1}=$ $g \in C\left(Y, K_{2}\right), g_{1} \in C\left(Y, K_{1}\right),(\mathcal{O}(f, u)(v))$ is defined on $(\hat{f}, \hat{u})\left(C\left(X, K_{1}\right)\right)=\left\{t: t \in C\left(Y, K_{2}\right) ; \forall x \in\right.$ $\left.X t(x)=u(h \circ f(x)), h \in C\left(Y, K_{1}\right)\right\}$.

By $I(f, u)$ will be denoted the restriction of $\mathcal{O}(f, u)$ onto $I(X, K)$. The shorter notations $\mathcal{O}(f)$ and $I(f)$ are used when $K$ is fixed, i.e. $u=i d$. When $X=Y$ and $f=i d$ we write simply $\mathcal{O}_{2}(u)$ and $I_{2}(u)$ respectively omitting $f=i d$. 
Let $\mathcal{S}$ denote a category such that a family $O b(\mathcal{S})$ of its objects consists of all topological spaces, a family of morphisms $\operatorname{Mor}(X, Y)$ consists of all continuous mappings $f: X \rightarrow Y$ for every $X$, $Y \in O b(\mathcal{S})$.

Let $\mathcal{K}$ be the category objects of which $O b(\mathcal{K})$ are all ordered topological ringoids satisfying Conditions 2.3 and 2.4, $\operatorname{Mor}(A, B)$ consists of all order-preserving continuous algebraic homomorphisms for each $A, B \in \mathcal{K}$. Then by $\mathcal{K}_{w}$ we denote its subcategory of well-ordered ringoids and their order-preserving algebraic continuous homomorphisms.

We denote by $\mathcal{O K}$ a category with the families of objects $O b(\mathcal{O K})=\{\mathcal{O}(X, K): X \in O b(\mathcal{S})$, $\left.K \in O b\left(\mathcal{K}_{w}\right)\right\}$ and morphisms $\operatorname{Mor}\left(\mathcal{O}\left(X, K_{1}\right), \mathcal{O}\left(Y, K_{2}\right)\right)$ for every $X, Y \in O b(\mathcal{S})$ and $K_{1}, K_{2} \in$ $\mathrm{Ob}\left(\mathcal{K}_{w}\right)$. Furthermore, $\mathcal{I} \mathcal{K}$ stands for a category with families of objects $\mathrm{Ob}(\mathcal{I} \mathcal{K})=\{I(X, K)$ : $\left.X \in O b(\mathcal{S}), K \in O b\left(\mathcal{K}_{w}\right)\right\}$ and morphisms $\operatorname{Mor}\left(I\left(X, K_{1}\right), I\left(Y, K_{2}\right)\right)$ for every $X, Y \in O b(\mathcal{S})$ and $K_{1}, K_{2} \in O b(\mathcal{K})$.

By $\mathcal{S}_{l}$ will be denoted a category objects of which are linearly ordered topological spaces, while $\operatorname{Mor}(X, Y)$ consists of all monotone nondecreasing continuous mappings $f: X \rightarrow Y$, that is $f(x) \leq f(y)$ for each $x \leq y \in X$, where $X, Y \in O b\left(\mathcal{S}_{l}\right)$. Then we put $\mathcal{O}_{l}(f, u): \mathcal{O}_{l}\left(X, K_{1}\right) \rightarrow \mathcal{O}_{l}\left(Y, K_{2}\right)$ for each $X, Y \in O b\left(\mathcal{S}_{l}\right)$ and $f \in \operatorname{Mor}(X, Y), K_{1}, K_{2} \in \operatorname{Ob}(\mathcal{K}), u \in \operatorname{Mor}\left(K_{1}, K_{2}\right)$ according to the formula:

(2) $\left(\mathcal{O}_{l}(f, u)(v)\right)(g)=u\left[v\left(g_{1}(f)\right)\right]$ for each $g_{1} \in C_{+}\left(Y, K_{1}\right)$ and $u \circ g_{1}=g \in C\left(Y, K_{2}\right)$ and $v \in \mathcal{O}_{l}\left(X, K_{1}\right)$, where $\left(\mathcal{O}_{l}(f, u)(v)\right)$ is defined on $(\hat{f}, \hat{u})\left(C_{+}\left(X, K_{1}\right)\right):=\left\{t: t \in C_{+}\left(Y, K_{2}\right)\right.$; $\left.\forall x \in X t(x)=u(h \circ f(x)), h \in C_{+}\left(Y, K_{1}\right)\right\}$. Then the category $\mathcal{O}_{l} \mathcal{K}$ with families of objects $\mathrm{Ob}\left(\mathcal{O}_{l} \mathcal{K}\right)=\left\{\mathcal{O}_{l}(X, K): X \in O b\left(\mathcal{S}_{l}\right), K \in O b(\mathcal{K})\right\}$ and morphisms $\operatorname{Mor}\left(\mathcal{O}_{l}\left(X, K_{1}\right), \mathcal{O}_{l}\left(Y, K_{2}\right)\right)$ and the category $\mathcal{I}_{l} \mathcal{K}$ with $\mathrm{Ob}\left(\mathcal{I}_{l} \mathcal{K}\right)=\left\{I_{l}(X, K): X \in O b\left(\mathcal{S}_{l}\right), K \in O b(\mathcal{K})\right\}$ and $\operatorname{Mor}\left(I_{l}\left(X, K_{1}\right), I_{l}\left(Y, K_{2}\right)\right)$ are defined.

Subcategories of left homogeneous continuous morphisms we denote by $\mathcal{O}_{h} \mathcal{K}, \mathcal{O}_{l, h} \mathcal{K}, \mathcal{I}_{h} \mathcal{K}, \mathcal{I}_{l, h} \mathcal{K}$ correspondingly. These morphisms are taken on subcategories $\mathcal{K}_{w, l}$ in $\mathcal{K}$ or $\mathcal{K}_{l}$ in $\mathcal{K}$ of left distributive topological ringoids.

21. Lemma. There exist covariant functors $\mathcal{O}, \mathcal{O}_{h}$ and $\mathcal{O}_{l}, \mathcal{O}_{l, h}$ in the categories $\mathcal{S}$ and $\mathcal{S}_{l}$ respectively.

Proof. Suppose that $X, Y \in O b(\mathcal{S})$ and $f \in \operatorname{Mor}(X, Y)$, while $g \leq h$ in $C(Y, K)$, where $K \in O b\left(\mathcal{K}_{w}\right)$ (or in $\mathcal{K}_{w, l}$ ) is marked, then $g \circ f \leq h \circ f$ in $C(X, Y)$. Therefore one gets $(\mathcal{O}(f)(v))(g)=$ $v(g \circ f) \leq v(h \circ f)=(\mathcal{O}(f)(v))(h)$ for each $v \in \mathcal{O}(X, K)$. Now if $c \in K, g^{c} \in C(Y, K)$, then $g^{c} \circ f \in C(X, K)$, but also the equalities are fulfilled $(\mathcal{O}(f)(v))\left(g^{c}+h\right)=v\left(g^{c} \circ f+h \circ f\right)=c+v(h \circ$ $f)=c+(\mathcal{O}(f)(v))(h)$ and $(\mathcal{O}(f)(v))\left(h+g^{c}\right)=v\left(h \circ f+g^{c} \circ f\right)=v(h \circ f)+c=(\mathcal{O}(f)(v))(h)+c$ for each $h \in C(Y, K)$. Then for $1_{X} \in \operatorname{Mor}(X, X)$, that is $1_{X}(x)=x$ for each $x \in X$, one deduces $1_{X} \circ q=q$ for each $q \in \operatorname{Mor}(Y, X)$ and $t \circ 1_{X}=t$ for each $t \in \operatorname{Mor}(X, Y)$. On the other hand, $\left(\mathcal{O}\left(1_{X}\right)(v)\right)(g)=v\left(g \circ 1_{X}\right)=v(g)$ for each $g \in C(X, K)$, i.e. $\mathcal{O}\left(1_{X}\right)=1_{\mathcal{O}(X)}$. But at the same time, the equalities are valid: $(\mathcal{O}(f \circ s)(v))(g)=v(g \circ f \circ s)=(\mathcal{O}(s)(v)(g \circ f)=((\mathcal{O}(f) \circ \mathcal{O}(s))(v))(g)$, since the composition of continuous mappings is continuous.

Moreover, if $v \in \mathcal{O}_{h}(X, K)$, then $(\mathcal{O}(f)(v))(b g)=v(b g \circ f)=b v(g \circ f)=(b(\mathcal{O}(f)(v))(g)$. Furthermore, for the categories $\mathcal{O}_{l}\left(\right.$ or $\left.\mathcal{O}_{l, h}\right)$ the proof is analogous with $X, Y \in O b\left(\mathcal{S}_{l}\right), C_{+}(X, K)$ and $C_{+}(Y, K)$, where $K \in O b(\mathcal{K})$ (or $K \in O b\left(\mathcal{K}_{l}\right)$ ) is marked.

22. Proposition. Suppose that $f \in \operatorname{Mor}(X, Y)$ for $X, Y \in O b(\mathcal{S})$ or in $\mathrm{Ob}\left(\mathcal{S}_{l}\right)$. Then

$\mathcal{O}(f)(I(X, K)) \subseteq I(Y, K)$ and $\mathcal{O}_{h}(f)\left(I_{h}(X, K)\right) \subseteq I_{h}(Y, K)$ for $K \in O b\left(\mathcal{K}_{w, l}\right)$ or $\mathcal{O}_{l}(f)\left(I_{h}(X, K)\right) \subseteq$ $I_{l}(Y, K)$ or $\mathcal{O}_{l, h}(f)\left(I_{l, h}(X, K)\right) \subseteq I_{l, h}(Y, K)$ for $K \in O b(\mathcal{K})$ or $K \in O b\left(\mathcal{K}_{l}\right)$ correspondingly.

Proof. If $g, h \in C(Y, K)$ are such that $g \vee h$ or $g \wedge h$ exists (see Condition (3) in Lemma 2.12) and $f: X \rightarrow Y$ is a continuous mapping, $v \in I(X, K)$ (or $I_{l}(X, K)$ ), then we infer that

$(\mathcal{O}(f)(v))(g \vee h)=v(g \circ f \vee h \circ f)=v(g \circ f) \vee v(h \circ f)=(\mathcal{O}(f)(v))(g) \vee(\mathcal{O}(f)(v))(h)$ or

$(\mathcal{O}(f)(v))(g \wedge h)=v(g \circ f \wedge h \circ f)=v(g \circ f) \wedge v(h \circ f)=(\mathcal{O}(f)(v))(g) \wedge(\mathcal{O}(f)(v))(h)$.

Furthermore, for each $c \in K$ we deduce that

$(\mathcal{O}(f)(v))\left(g^{c} \times{ }_{2} h\right)=v\left(g^{c} \circ f \times_{2} h \circ f\right)=v\left(g^{c} \circ f\right) \times_{2} v(h \circ f)=c \times_{2}(\mathcal{O}(f)(v))(h)$ and

$(\mathcal{O}(f)(v))\left(h \times_{2} g^{c}\right)=v\left(h \circ f \times_{2} g^{c} \circ f\right)=v(h \circ f) \times_{2} v\left(g^{c} \circ f\right)=(\mathcal{O}(f)(v))(h) \times_{2} c$. 
Then for $v \in I_{h}(X, K)\left(\right.$ or $\left.I_{l, h}(X, K)\right)$ one gets $(\mathcal{O}(f)(v))(b g)=v(b g \circ f)=b v(g \circ f)=$ $(b(\mathcal{O}(f)(v)))(g)$.

23. Definitions. A covariant functor $F: \mathcal{S} \rightarrow \mathcal{S}$ will be called epimorphic (monomorphic) if it preserves continuous epimorphisms (monomorphisms). If $\phi: A \hookrightarrow X$ is a continuous embedding, then $F(A)$ will be identified with $F(\phi)(F(A))$.

If for each $f \in \operatorname{Mor}(X, Y)$ and each closed subset $A$ in $Y$, the equality $\left(F(f)^{-1}\right)(F(A))=$ $F\left(f^{-1}(A)\right)$ is satisfied, then a covariant functor $F$ is called preimage-preserving. In the case $F\left(\bigcap_{j \in J} X_{j}\right)=\bigcap_{j \in J} F\left(X_{j}\right)$ for each family $\left\{X_{j}: j \in J\right\}$ of closed subsets in $X \in O b(\mathcal{S})$ (or in $\left.O b\left(\mathcal{S}_{l}\right)\right)$, the monomorphic functor $F$ is called intersection-preserving.

If a functor $F$ preserves inverse mapping system limits, it is called continuous.

A functor is said to be semi-normal when it is monomorphic, epimorphic, also preserves intersections, preimages and the empty space.

If a functor is monomorphic, epimorphic, also preserves intersections and the empty space, then it is called weakly semi-normal.

24. Proposition. The functor $\mathcal{O}$ (or $\mathcal{O}_{h}, \mathcal{O}_{l}, \mathcal{O}_{l, h}$ ) is monomorphic.

Proof. Let $X, Y \in O b(\mathcal{S})$ (or in $O b\left(\mathcal{S}_{l}\right)$ respectively) with a continuous embedding $s: X \hookrightarrow Y$ (order-preserving respectively). Then we suppose that $v_{1} \neq v_{2} \in \mathcal{O}(X, K)$ (or in $\mathcal{O}_{h}(X, K)$, $\mathcal{O}_{l}(X, K), \mathcal{O}_{l, h}(X, K)$ correspondingly). This means that a mapping $g \in C(X, K)$ (or in $C_{+}(X, K)$ correspondingly) exists such that $v_{1}(g) \neq v_{2}(g)$. A function $u \in C(Y, K)$ (or in $C_{+}(Y, K)$ respectively) exists such that $u \circ s=g$, hence $\left.\left(\mathcal{O}(s)\left(v_{k}\right)\right)\right)(u)=v_{k}(u \circ s)=v_{k}(g)$. Thus $\mathcal{O}(s)\left(v_{1}\right) \neq \mathcal{O}(s)\left(v_{2}\right)$ (or $\mathcal{O}_{h}\left(v_{1}\right) \neq \mathcal{O}_{h}\left(v_{2}\right), \mathcal{O}_{l}\left(v_{1}\right) \neq \mathcal{O}_{l}\left(v_{2}\right), \mathcal{O}_{l, h}\left(v_{1}\right) \neq \mathcal{O}_{l, h}\left(v_{2}\right)$ correspondingly $)$.

25. Corollary. The functors $I, I_{h}, I_{l}$ and $I_{l, h}$ are monomorphic.

Proof. This follows from Proposition 24 and Definitions 20.

26. Proposition. The functors $\mathcal{O}, \mathcal{O}_{h}, \mathcal{O}_{l}$ and $\mathcal{O}_{l, h}$ are epimorphic, when $X \in H_{X}$ (see $\S 14$ also).

Proof. Let $f: X \rightarrow Y$ be a continuous surjective mapping, $v \in \mathcal{O}(Y, K)$ (or in $\mathcal{O}_{h}(Y, K), \mathcal{O}_{l}(Y, K)$, $\mathcal{O}_{l, h}(Y, K)$ respectively). The set $L$ of all continuous mappings $g \circ f: X \rightarrow K$ with $g \in C(Y, K)$ (or in $C_{+}(Y, K)$ correspondingly) is the $A$-subset according to Definitions 6 or the left module over $K$ in $C(X, K)$ (or in $\left.C_{+}(X, K)\right)$. Then we put $\mu(g \circ f)=v(g)$. This continuous morphism has an extension from $L$ to a continuous morphism $\mu \in \mathcal{O}(X, K)$ (or in $\mathcal{O}_{h}(X, K), \mathcal{O}_{l}(X, K), \mathcal{O}_{l, h}(X, K)$ correspondingly) due to Lemmas 9, 14 and Corollary 8.

27. Lemma. Let $L$ be a submodule over $K$ of $C(X, K)$ or $C_{+}(X, K)$ relative to the operations $\vee, \wedge, \times_{2}$ and containing all constant mappings $g^{c}: X \rightarrow K$, where $c \in K$. Let also $v: L \rightarrow K$ be an idempotent (left homogeneous) continuous morphism. For each $f \in C(X, K) \backslash L$ or $C_{+}(X, K) \backslash L$ there exists an idempotent (left homogeneous) continuous extension $\mu_{M}$ of $v$ on a minimal closed submodule $M$ containing $L$ and $f$.

Proof. For each $g \in M$ we put

(1) $\mu_{M}(g)=v(g)=\inf \{v(h): g \leq h, h \in L\}$.

This implies that $v\left(g_{1}\right) \leq v\left(g_{2}\right)$ for each $g_{1} \leq g_{2} \in M$. Then

$v\left(g^{c} \times_{2} g\right)=\inf \left\{v(h): h \in L, g^{c} \times 2 g \leq h\right\}=$

$\inf \left\{v\left(g^{c} \times{ }_{2} q\right): q \in L, g^{c} \times 2 g \leq g^{c} \times{ }_{2} q\right\}=c \times_{2} \inf \{v(q): q \in L, g \leq q\}=c \times_{2} v(g)$ and

$v\left(g \times 2 g^{c}\right)=\inf \left\{v(h): h \in L, g \times 2 g^{c} \leq h\right\}=\inf \left\{v\left(q \times 2 g^{c}\right): q \in L, q \times 2 g^{c} \geq g \times 2 g^{c}\right\}$

$=\inf \{v(q): q \in L, q \geq g\} \times{ }_{2} c=v(g) \times{ }_{2} c$.

On the other hand for each $g_{1}, g_{2} \in M$ one gets

$v\left(g_{1}\right) \vee v\left(g_{2}\right)=\inf \left\{v(g): g \in L, g_{1} \leq g\right\} \vee \inf \left\{v(q): q \in L, g_{2} \leq q\right\}$

$=\inf \left\{v(g) \vee v(q): g, q \in L, g_{1} \leq g_{1}, g_{2} \leq q\right\} \geq \inf \left\{v(g \vee q): g, q \in L, g_{1} \vee g_{2} \leq g \vee q\right\}=v\left(g_{1} \vee g_{2}\right)$.

From the inequalities $g_{k} \leq g_{1} \vee g_{2}$ for $k=1$ and $k=2$ it follows, that $v\left(g_{k}\right) \leq v\left(g_{1} \vee g_{2}\right)$, consequently, $v\left(g_{1}\right) \vee v\left(g_{2}\right)=v\left(g_{1} \vee g_{2}\right)$. Then

$v\left(g_{1}\right) \wedge v\left(g_{2}\right)=\inf \left\{v(g): g \in L, g_{1} \leq g\right\} \wedge \inf \left\{v(q): q \in L, g_{2} \leq q\right\}$

$=\inf \left\{v(g) \wedge v(q): g, q \in L, g_{1} \leq g, g_{2} \leq q\right\} \leq \inf \left\{v(g \wedge q): g, q \in L, g_{1} \wedge g_{2} \leq g \wedge q\right\}=v\left(g_{1} \wedge g_{2}\right)$.

But $v\left(g_{k}\right) \geq v\left(g_{1} \wedge g_{2}\right)$, since $g_{k} \geq g_{1} \wedge g_{2}$ for $k=1$ and $k=2$, consequently, $v\left(g_{1}\right) \wedge v\left(g_{2}\right)=$ $v\left(g_{1} \wedge g_{2}\right)$. If $v$ is left homogeneous, then $\inf \{v(b h): b h \geq b g, h \in L\}=\inf \{v(b h): h \geq g, h \in$ 
$L\}=b \inf \{v(h): h \geq g, h \in L\}$ for each $b \in K$, consequently, $v$ is left homogeneous on $M$. If $v$ is continuous and $g_{k}$ is a net in $M$ converging to $g \in M$ (see \$2.9), then $v(g)=\inf \{v(h): g \leq h, h \in$ $L\}=\lim _{k} \inf \left\{v(h): g_{k} \leq h, h \in L\right\}=\lim _{k} v\left(g_{k}\right)$.

28. Lemma. If suppositions of Lemma 27 are satisfied, then there exists an idempotent (left homogeneous) continuous morphism $\lambda$ on $C(X, K)$ or $C_{+}(X, K)$ respectively such that $\left.\lambda\right|_{L}=v$.

Proof. The family of all extensions $\left(M, \mu_{M}\right)$ of $v$ on closed submodules $M$ of $C(X, K)$ or $C_{+}(X, K)$ respectively is partially ordered by inclusion: $\left(M, \mu_{M}\right) \leq\left(N, \mu_{N}\right)$ if and only if $M \subset N$ and $\left.v_{N}\right|_{M}=v_{M}$. In view of the Kuratowski-Zorn lemma [20] there exists the maximal closed submodule $P$ in $C(X, K)$ or $C_{+}(X, K)$ correspondingly and an idempotent extension $v_{P}$ of $v$ on $P$. If $P \neq C(X, K)$ or $C_{+}(X, K)$ correspondingly by Lemma 27 this morphism $v_{P}$ could be extended on a module $L$ containing $P$ and some $g \in C(X, K) \backslash P$ or in $C(X, K)_{+} \backslash P$ respectively. This contradicts the maximality of $\left(P, v_{P}\right)$. Thus $P=C(X, K)$ or $C_{+}(X, K)$ correspondingly.

29. Proposition. The functors $I, I_{l}$ and $I_{h}, I_{l, h}$ are epimorphic.

Proof. Let a continuous mapping $f: X \rightarrow Y$ be epimorphic. We consider the set $L$ of all continuous mappings $g \circ f: X \rightarrow K$ such that $g \in C(Y, K)$ or $C_{+}(Y, K)$. Then $L$ is a submodule of $C(X, K)$ or $C_{+}(X, K)$ relative to the operations $\vee, \wedge, \times_{2}$ and $L$ contains all constant mappings $g^{c}: X \rightarrow K$, where $c \in K$. Then we put $\mu(g \circ f)=v(g)$ for $v \in I(X, K)$ or in $I_{l}(X, K), I_{h}(X, K)$ or $I_{l, h}(X, K)$. In view of Lemma 28 there is a continuous extension of $\mu$ from $L$ onto $C(Y, K)$ or $C_{+}(Y, K)$ such that $\mu \in I(Y, K)$ or in $I_{l}(Y, K), I_{h}(Y, K)$ or $I_{l, h}(Y, K)$ correspondingly.

30. Definition. It is said that $v \in \mathcal{O}(X, K)$ (or $v \in \mathcal{O}_{l}(X, K)$ ) is supported on a closed subset $E$ in $X$, if $v(f)=0$ for each $f \in C(X, K)$ or in $C_{+}(X, K)$ such that $\left.f\right|_{E} \equiv 0$. A support of $v$ is the intersection of all closed subsets in $X$ on which $v$ is supported.

31. Proposition. Let $v \in \mathcal{O}(X, K)$ or in $\mathcal{O}_{l}(X, K)$. Then $v$ is supported on $E \subset X$ if and only if $v(f)=v(g)$ for each $f, g \in C(X, K)$ or in $C_{+}(X, K)$ correspondingly such that $\left.\left.f\right|_{E} \equiv g\right|_{E}$. Moreover, $E$ is a support of $v$ if and only if $v$ is supported on $E$ and for each proper closed subset $F$ in $E$, i.e. $F \subset E$ with $F \neq E$, there are $f, h \in C(X, K)$ or in $C_{+}(X, K)$ respectively with $\left.\left.f\right|_{F} \equiv h\right|_{F}$ such that $v(f) \neq v(h)$.

Proof. Consider $v \in \mathcal{O}(X, K)$ such that $v(f)=v(g)$ for each functions $f, g: X \rightarrow K$ with $\left.f\right|_{E}=\left.g\right|_{E}$. A continuous morphism $v$ induces a continuous morphism $\lambda \in \mathcal{O}(E, K)$ such that $\lambda(h)=v(h)$ for each $h \in C(X, K)$ with $\left.h\right|_{X \backslash E}=0$. Denote by $i d$ the identity embedding of a closed subset $E$ into $X$. Each function $t: E \rightarrow K$ has an extension on $X$ with values in $K$ by Condition $19(C E)$. Then $\mathcal{O}(i d)(\lambda)=v$, since $v\left(g^{0}\right)=0$ and hence $v(s)=0$ for each $s \in C(X, K)$ such that $\left.s\right|_{E} \equiv 0$.

If $v \in \mathcal{O}(X, K)$ and $v$ is supported on $E$, then by Definition 30 there exists a morphism $\lambda \in \mathcal{O}(E, K)$ such that $\mathcal{O}(i d)(\lambda)=v$. Therefore the equalities are valid: $v(f)=\lambda\left(\left.f\right|_{E}\right)=\lambda\left(\left.g\right|_{E}\right)=$ $v(g)$ for each functions $f, g \in C(X, K)$ such that $\left.f\right|_{E}=\left.g\right|_{E}$.

If $E$ is a support of $v$, then by the definition this implies that $v$ is supported on $E$. Suppose that $F \subset E, F \neq E$ and for each $f, g \in C(X, K)$ with $\left.\left.f\right|_{F} \equiv g\right|_{F}$ the equality $v(f)=v(g)$ is satisfied, then a support of $v$ is contained in $F$, hence $E$ is not a support of $v$. This is the contradiction, hence there are $f, g \in C(X, K)$ with $\left.\left.f\right|_{F} \equiv g\right|_{F}$ such that $v(f) \neq v(g)$.

If $v$ is supported on $E$ and for each proper closed subset $F$ in $E$ there are $f, h \in C(X, K)$ with $\left.\left.f\right|_{F} \equiv h\right|_{F}$ such that $v(f) \neq v(h)$, then $v$ is not supported on any such proper closed subset $F$, consequently, each closed subset $G$ in $X$ on which $v$ is supported contains $E$, i.e. $E \subset G$. Thus $E$ is the support of $v$.

32. Proposition. The functors $\mathcal{O}, I, \mathcal{O}_{l}, I_{l}, \mathcal{O}_{l, h}, I_{l, h}$ preserve intersections of closed subsets.

Proof. If $E$ is a closed subset in $X$, then there is the natural embedding $C(E, K) \hookrightarrow C(X, K)$ (or $C_{+}(E, K) \hookrightarrow C_{+}(X, K)$, when $\left.X \in O b\left(\mathcal{S}_{l}\right)\right)$ due to Condition 19(CE). Therefore, $\mathcal{O}(E \cap F, K) \subset$ $\mathcal{O}(E, K) \cap \mathcal{O}(F, K)$ (or $\mathcal{O}_{l}(E \cap F, K) \subset \mathcal{O}_{l}(E, K) \cap \mathcal{O}_{l}(F, K)$ respectively). For any closed subsets $E$ and $F$ in $X$ and each functions $f, g \in C(X, K)$ (or $C_{+}(X, K)$ ) with $\left.\left.f\right|_{E \cap F} \equiv g\right|_{E \cap F}$ there exists a function $h \in C(X, K)$ (or $C_{+}(X, K)$ ) such that $\left.h\right|_{E}=f$ and $\left.h\right|_{F}=g$ due to $19(C E)$. Therefore $v(f)=v(h)$ and $v(g)=v(h)$ for each $v \in \mathcal{O}(E, K) \cap \mathcal{O}(F, K)$ (or in $\mathcal{O}_{l}(E, K) \cap \mathcal{O}_{l}(F, K)$ ). In view of Proposition 31 the 
functors $\mathcal{O}$ and $\mathcal{O}_{l}$ preserve intersections of closed subsets. This implies that the functors $I, I_{l}, \mathcal{O}_{l, h}$ and $I_{l, h}$ also have this property.

33. Proposition. Let $\left\{X_{b} ; p_{a}^{b} ; V\right\}=: P$ be an inverse system of topological spaces $X_{b}$, where $V$ is $a$ directed set, $p_{a}^{b}: X_{b} \rightarrow X_{a}$ is a continuous mapping for each $a \leq b \in V, p_{b}: X=\lim P \rightarrow X_{b}$ is a continuous projection. Then the mappings

(1) $s=\left(\mathcal{O}\left(p_{b}\right): b \in V\right): \mathcal{O}(X, K) \rightarrow \mathcal{O}(P, K)$ and $s_{h}=\left(\mathcal{O}_{h}\left(p_{b}\right): b \in V\right): \mathcal{O}_{h}(X, K) \rightarrow \mathcal{O}_{h}(P, K)$

(2) $t=\left(I\left(p_{b}\right): b \in V\right): I(X, K) \rightarrow I(P, K)$ and $t_{h}=\left(I_{h}\left(p_{b}\right): b \in V\right): I_{h}(X, K) \rightarrow I_{h}(P, K)$

are bijective and surjective continuous algebraic homomorphisms. Moreover, if $X_{b} \in \mathrm{Ob}\left(\mathcal{S}_{l}\right)$ and $p_{a}^{b}$ is order-preserving for each $a<b \in V$, then the mappings

(3) $s_{l}=\left(\mathcal{O}_{l}\left(p_{b}\right): b \in V\right): \mathcal{O}_{l}(X, K) \rightarrow \mathcal{O}_{l}(P, K)$ and $s_{l, h}=\left(\mathcal{O}_{l, h}\left(p_{b}\right): b \in V\right): \mathcal{O}_{l, h}(X, K) \rightarrow$ $\mathcal{O}_{l, h}(P, K)$

(4) $t_{l}=\left(I_{l}\left(p_{b}\right): b \in V\right): I_{l}(X, K) \rightarrow I_{l}(P, K)$ and $t_{l, h}=\left(I_{l, h}\left(p_{b}\right): b \in V\right): I_{l, h}(X, K) \rightarrow I_{l, h}(P, K)$ also are bijective and surjective continuous algebraic homomorphisms.

Proof. We consider the inverse system $\mathcal{O}(P)=\left(\mathcal{O}\left(X_{a}\right) ; \mathcal{O}\left(p_{b}^{a}\right) ; V\right\}$ and its limit space $Y=\lim \mathcal{O}(P)$. Then $\mathcal{O}\left(p_{a}^{b}\right) \mathcal{O}\left(p_{b}\right)=\mathcal{O}\left(p_{a}\right)$ for each $a \leq b \in V$, since $p_{a}^{b} \circ p_{b}=p_{a}$. Let $q: \mathcal{O}(X, K) \rightarrow Y$ denote the limit map of the inverse mapping system $q=\lim \left\{\mathcal{O}\left(p_{a}\right) ; \mathcal{O}\left(p_{b}^{a}\right) ; V\right\}$ (see also $\$ 2.5[21]$ ).

A continuous morphism $v$ is in $\mathcal{O}(X, K)$ if and only if $\mathcal{O}\left(p_{a}\right)(v) \in \mathcal{O}\left(X_{a}, K\right)$ for each $a \in V$, since

(5) $f \in C(X, K)$ if and only if $f=\lim \left\{f_{b} ; p_{a}^{b} ; V\right\}$ and

(6) $\mathcal{O}\left(p_{a}\right)(v)\left(f_{a}\right)=v\left(f_{a} \circ p_{a}\right)=v_{a}\left(f_{a}\right)$, where $v_{a} \in \mathcal{O}\left(X_{a}, K\right), f_{b} \in C\left(X_{b}, K\right), f_{b}=f_{a} \circ p_{a}^{b}$ for each $a \leq b \in V, p_{b}^{b}=i d, f(x)=\left\{f_{a} \circ p_{a}(x): a \in V\right\} \in \theta(K)$ for each $x=\left\{x_{a}: a \in V\right\} \in X$, where $\left\{x_{a}: a \in V\right\}$ is a thread of $P$ such that $x_{a} \in X_{a}, p_{a}^{b}\left(x_{b}\right)=x_{a}$ for each $a \leq b \in V, \theta: K \rightarrow K^{X}$ is an order-preserving continuous algebraic embedding, $\theta(K)$ is isomorphic with $K$.

If $v, \lambda \in \mathcal{O}(X, K)$ are two different continuous morphisms, then this means that a continuous function $f \in C(X, K)$ exists such that $v_{1}(f) \neq v_{2}(f)$. This is equivalent to the following: there exists $a \in V$ such that $\left(\mathcal{O}\left(p_{a}\right)(v)\right)(f) \neq\left(\mathcal{O}\left(p_{a}\right)(\lambda)\right)(f)$. Thus the mappings $s$ and analogously $t$ are surjective and bijective.

On the other hand,

(7) $v_{b}\left(f_{b} \vee g_{b}\right)=v_{b}\left(f_{b}\right) \vee v_{b}\left(g_{b}\right)$ and

(8) $v_{b}\left(f_{b} \wedge g_{b}\right)=v_{b}\left(f_{b}\right) \wedge v_{b}\left(g_{b}\right)$ for each $b \in V$ and each $v_{b} \in I\left(X_{b}, K\right)$ and every $f_{b}, g_{b} \in$ $C\left(X_{b}, K\right)$ such that either $f_{b}(x)<g_{b}(x)$ or $f_{b}(x)=g_{b}(x)$ or $g_{b}(x)<f_{b}(x)$ for each $x \in X_{b}$, also

(9) $v_{b}\left(g^{c} \times_{2} f_{b}\right)=c \times_{2} v_{b}\left(f_{b}\right)$ and

(10) $v_{b}\left(f_{b} \times{ }_{2} g^{c}\right)=v_{b}\left(f_{b}\right) \times_{2} c$ for each $c \in K$ and $f_{b} \in C\left(X_{b}, K\right)$. Taking the inverse limit in Equalities $(5-10)$ gives the corresponding equalities for $v \in I(X, K)$, where $v=\lim \left\{v_{a} ;\left(p_{a}^{b}\right) ; V\right\}$, hence $t$ is the continuous algebraic homomorphism due to Theorem 2.5.8 [21].

Analogously s preserves Properties $(9,10)$, that is $\lambda=\lim \left\{\lambda_{a} ; \mathcal{O}\left(p_{a}^{b}\right) ; V\right\}$ is weakly additive, where $\lambda_{b} \in \mathcal{O}\left(X_{b}, K\right)$ for each $b \in V$. Suppose that $f \leq g \in C(X, K)$, then $f_{b} \leq g_{b}$ for each $b \in V$ due to (5). From $\lambda_{b}\left(f_{b}\right) \leq \lambda_{b}\left(g_{b}\right)$ for each $b \in V$, the inverse limit decomposition $\lambda=\lim \left\{\lambda_{b} ; \mathcal{O}\left(p_{a}^{b}\right) ; V\right\}$ and Formula (6) it follows that $\lambda$ is order-preserving.

If $X_{b} \in O b\left(\mathcal{S}_{l}\right)$ for each $b \in V$, then a topological space $X$ is linearly ordered: $x=\left\{x_{b}: b \in V\right\} \leq$ $y=\left\{y_{b}: b \in V\right\}$ if and only if $x_{b} \leq y_{b}$ for each $b \in V$, where $x, y \in X$ are threads of the inverse system $P$ such that $p_{a}^{b}\left(x_{b}\right)=x_{a}$ for each $a \leq b \in V$. Since $p_{a}^{b}$ is order-preserving for each $a \leq b \in V$ and each $f_{b}$ is non-decreasing, then $f$ is nondecreasing and hence $f \in C_{+}(X, K)$ for each $f=\lim \left\{f_{b} ; p_{a}^{b} ; V\right\}$, where $f_{b} \in C_{+}\left(X_{b}, K\right)$ and $f_{b}=f_{a} \circ p_{a}^{b}$ for each $a \leq b \in V$ and $x \in X, f(x)=\left\{f_{a} \circ p_{a}(x): a \in V\right\}$.

Moreover, $v \in \mathcal{O}_{h}(X, K)$ is left homogeneous if and only if $\theta\left(p_{a}\right)(v)$ is left homogeneous for each $b \in V$, since $\left(\mathcal{O}_{h}\left(p_{a}\right)(v)\right)\left(f_{a}\right)=v\left(f_{a} \circ p_{a}\right)=v_{a}\left(f_{a}\right)$. Applying Lemma 2.5.9 [21] one gets properties of mappings in Formulas $(3,4)$. 
34. Lemma. There exist covariant functors $\mathcal{O}_{2}, I_{2}$, and $\mathcal{O}_{l, 2}, I_{l, 2}$ and $\mathcal{O}_{h, 2}, I_{h, 2}$ and $\mathcal{O}_{l, h, 2}, I_{l, h, 2}$ in the categories $\mathcal{K}_{w}$ and $\mathcal{K}$ and $\mathcal{K}_{w, l}$ and $\mathcal{K}_{l}$ respectively.

Proof. If $K_{1}, K_{2}, K_{3} \in \operatorname{Ob}\left(\mathcal{K}_{w}\right), u \in \operatorname{Mor}\left(K_{1}, K_{2}\right), v \in \operatorname{Mor}\left(K_{2}, K_{3}\right), v \in I\left(X, K_{1}\right)$, then $\left(I_{2}(v u)(v)\right)(f)=v \circ u \circ v\left(f_{1}\right)=\left[I_{2}(v)\left(I_{2}(u)(v)\right)\right](f)$ for each $f_{1} \in C\left(X, K_{1}\right)$ such that $f(x)=v \circ u \circ f_{1}(x)$ for each $x \in X$, where $X \in O b(\mathcal{S})$. That is $I_{2}(v u)=I_{2}(v) I_{2}(u)$. On the other hand, the equality $I_{2}(i d)=1$ is fulfilled.

If $f(x) \leq g(x)$, then $u(f(x)) \leq u(g(x))$, where $x \in X, f, g \in C\left(X, K_{1}\right)$. Therefore, if a mapping either $f \vee g$ or $f \wedge g$ exists in $C\left(X, K_{1}\right)$, then $u(f \vee g)=u(f) \vee u(g)$ or $u(f \wedge g)=u(f) \wedge u(g)$ in $C\left(X, K_{2}\right)$ respectively. If $f, g \in C\left(X, K_{1}\right)$, then $u(f(x)+g(x))=u(f(x))+u(g(x))$ for each $x \in X$, particularly, this is valid for $f=g^{c}$ or $g=g^{c}$, where $c \in K_{1}$. Therefore, $u\left(g^{c} \times_{2} g\right)=g^{u(c)} \times{ }_{2} u(g)$ and $u\left(g \times{ }_{2} g^{c}\right)=u(g) \times 2 g^{u(c)}$. To each $v_{n} \in \mathcal{O}\left(X, K_{n}\right)$ and $u \in \operatorname{Mor}\left(K_{n}, K_{n+1}\right)$ there corresponds a morphism $u \circ v_{n}$ on $(\hat{i d}, \hat{u})\left(C\left(X, K_{n}\right)\right)$, $(\hat{i d}, \hat{u})\left(C\left(X, K_{n}\right)\right) \hookrightarrow C\left(X, K_{n+1}\right)$ (see §20). If $u: K_{n} \rightarrow K_{n+1}$ is not an epimorphism, the image $(\hat{i d}, \hat{u})\left(C\left(X, K_{n}\right)\right)$ is a proper submodule over $u\left(K_{n}\right)$ in $C\left(X, K_{n+1}\right)$.

If $K_{n}, K_{n+1} \in O b(\mathcal{K})$ and $X \in O b\left(\mathcal{S}_{l}\right), u \in \operatorname{Mor}\left(K_{n}, K_{n+1}\right)$, then $\hat{u}: C_{+}\left(X, K_{n}\right) \rightarrow C_{+}\left(X, K_{n+1}\right)$ is a continuous homomorphism. If $K_{n}, K_{n+1} \in O b\left(\mathcal{K}_{l}\right)$ and $X \in O b\left(\mathcal{S}_{l}\right)$ (or $K_{n}, K_{n+1} \in O b\left(\mathcal{K}_{w, l}\right)$ and $X \in O b(\mathcal{S}))$ and $v \in \mathcal{O}_{h}\left(X, K_{n}\right)$ or in $I_{h}\left(X, K_{n}\right), u \in \operatorname{Mor}\left(K_{n}, K_{n+1}\right)$, then $u \circ v \in \mathcal{O}_{h}\left(X, K_{n+1}\right)$ or in $I_{h}\left(X, K_{n+1}\right)$ respectively.

This and the definitions above imply that $\mathcal{O}_{2}(u): \mathcal{O}\left(X, K_{1}\right) \rightarrow \mathcal{O}\left(X, K_{2}\right), I_{2}(u): I\left(X, K_{1}\right) \rightarrow$ $I\left(X, K_{2}\right)$ and $\mathcal{O}_{l, 2}(u), I_{l, 2}(u)$ and $\mathcal{O}_{h, 2}(u), I_{h, 2}(u)$ and $\mathcal{O}_{l, h, 2}(u), I_{l, h, 2}(u)$ are the homomorphisms. Thus we deduce that $\mathcal{O}_{2}: \mathcal{K}_{w} \rightarrow \mathcal{O} \mathcal{K}$ and $\mathcal{O}_{l, 2}: \mathcal{K} \rightarrow \mathcal{O}_{l} \mathcal{K}, I_{2}: \mathcal{K}_{w} \rightarrow \mathcal{I} \mathcal{K}$ and $I_{l, 2}: \mathcal{K} \rightarrow \mathcal{I}_{l} \mathcal{K}, \mathcal{O}_{h, 2}: \mathcal{K}_{w, l} \rightarrow$ $\mathcal{O}_{h} \mathcal{K}, I_{h, 2}: \mathcal{K}_{w, l} \rightarrow \mathcal{I}_{h} \mathcal{K}, \mathcal{O}_{l, h, 2}: \mathcal{K}_{l} \rightarrow \mathcal{O}_{l, h} \mathcal{K}$ and $\mathcal{I}_{l, h, 2}: \mathcal{K}_{l} \rightarrow \mathcal{I}_{l, h} \mathcal{K}$ are the covariant functors on the categories $\mathcal{K}_{w}, \mathcal{K}, \mathcal{K}_{w, l}$ and $\mathcal{K}_{l}$ correspondingly with values in the categories of skew idempotent continuous morphisms, when a set $X \in O b(\mathcal{S})$ or in $\mathrm{Ob}\left(\mathcal{S}_{l}\right)$ correspondingly is marked.

35. Proposition. The bi-functors $I$ on $\mathcal{S} \times \mathcal{K}_{w}$, $I_{l}$ on $\mathcal{S}_{l} \times \mathcal{K}, I_{h}$ on $\mathcal{S} \times \mathcal{K}_{w, l}$ and $I_{l, h}$ on $\mathcal{S}_{l} \times \mathcal{K}_{l}$ preserve pre-images.

Proof. In view of Proposition 24 and Lemma $34 I, I_{l}, I_{h}$ and $I_{l, h}$ are the covariant bi-functors, i.e., the functors in $\mathcal{S}$ or $\mathcal{S}_{l}$ and the functors in $\mathcal{K}_{w}$ or $\mathcal{K}$ or $\mathcal{K}_{w, l}$ or $\mathcal{K}_{l}$ correspondingly as well. For any functor $F$ the inclusion $F\left(f^{-1}(B)\right) \subset(F(f))^{-1}(F(B))$ is satisfied, where, for example, $B$ is closed in $Y \in O b(\mathcal{S})$.

Suppose the contrary that $I$ does not preserve pre-images. This means that there exist $X, Y \in$ $\mathrm{Ob}(\mathcal{S})$ and $K_{1}, K_{2} \in \mathrm{Ob}\left(\mathcal{K}_{w}\right)$ or $X, Y \in O b\left(\mathcal{S}_{l}\right)$ and $K_{1}, K_{2} \in O b(\mathcal{K}), f \in \operatorname{Mor}(X, Y)$, u $\in \operatorname{Mor}\left(K_{1}, K_{2}\right)$, $A \subset X$ and $B \subset Y$, where $B$ is closed and hence $A$ is closed when $A=F^{-1}(B), v \in I\left(X, K_{1}\right)$ such that $I(f, u)(v) \in I\left(B, K_{2}\right)$ but $v \notin I\left(f^{-1}(B), u^{-1}\left(K_{2}\right)\right)$ (or $v \in I_{l}\left(X, K_{1}\right), I_{l}(f, u)(v) \in I_{l}\left(B, K_{2}\right)$ and $v \notin I_{l}\left(f^{-1}(B), u^{-1}\left(K_{2}\right)\right)$ respectively). One can choose two functions $g, h \in C\left(X, K_{1}\right)$ such that

(1) $\left.g\right|_{A}=\left.h\right|_{A}$

(2) $0<c_{1}=u\left[\inf _{x \in X} g(x)\right], 0<c_{2}=u\left[\inf _{x \in X} h(x)\right]$ and

(3) $u[v(g)] \neq u[v(h)]$.

There exist functions $s, t \in C\left(X, K_{1}\right)$ such that

(4) $\left.s\right|_{A}=\left.g\right|_{A}$ and $\left.t\right|_{A}=\left.h\right|_{A}$, while

(5) $\left.s\right|_{X \backslash A}=\left.t\right|_{X \backslash A}$ and

(6) $s(x) \leq g(x)$ and $s(x) \leq h(x)$ for each $x \in X \backslash A$, where $g$, $h$ satisfy Conditions $(1-3)$ due to property $19(C E)$. There are also functions $q, r \in C\left(X, K_{1}\right)$ such that

(7) $\left.q\right|_{X \backslash A}=\left.g\right|_{X \backslash A}$ and $\left.r\right|_{X \backslash A}=\left.h\right|_{X \backslash A}$ with

(8) $q(x)=r(x)$ and $q(x) \leq c$ for each $x \in A$, where

(9) $c \in K_{1}, c<\inf _{x \in X} g(x), c<\inf _{x \in X} h(x)$ such that $u(c)<c_{1}$ and $u(c)<c_{2}$.

Evidently, $c_{1} \leq u[v(g)]$ and $c_{2} \leq u[v(h)]$. Then

(10) $v(g)=v(s \vee q)=v(s) \vee v(q)$ and

(11) $v(h)=v(t \vee r)=v(t) \vee v(r)$ and $u[v(q)] \neq u[v(r)]$. 
On the other hand, there are functions $q_{1}, r_{1} \in C\left(Y, K_{2}\right), q_{2}, r_{2} \in C\left(Y, K_{1}\right)$ such that $q_{2} \circ f=q$, $r_{2} \circ f=r, u \circ q_{2}=q_{1}, u \circ r_{2}=r_{1}$ and $\left.q_{2}\right|_{B}=\left.r_{2}\right|_{B}$. Therefore, from Properties $(7-10)$ it follows that

(12) $(I(f, u)(v))\left(q_{1}\right)=u[v(q)] \leq u(c)$ and $(I(f, u)(v))\left(r_{1}\right)=u[v(r)] \leq u(c)$. The condition $s=t$ on $A$ and on $X \backslash A$ imply that

(13) $v(s)=v(t)$. Therefore,

(14) $u(v(g))=u(v(s)) \vee u(v(q))$ and $u(v(h))=u(v(t)) \vee u(v(r))$, which follows from $(10,11)$. But Formulas $(4-6,12-14)$ contradict the inequality $u[v(g)] \neq u[v(h)]$, since $u$ is the order-preserving continuous algebraic homomorphism from $K_{1}$ into $K_{2}$. Thus the bi-functors $I$ and $I_{l}$ preserve pre-images. The proof in other cases is analogous.

36. Corollary. If $v \in I(X, K)$ or $v \in I_{l}(X, K), f \in \operatorname{Mor}(X, Y), u \in \operatorname{Mor}\left(K_{1}, K_{2}\right)$, where $X, Y \in O b(\mathcal{S})$ and $K_{1}, K_{2} \in O b\left(\mathcal{K}_{w}\right)$ or $X, Y \in O b\left(\mathcal{S}_{l}\right)$ and $K_{1}, K_{2} \in O b(\mathcal{K})$, then $\operatorname{supp}(I(f, u)(v))=$ $f(\operatorname{supp}(u[v]))$ or

$\operatorname{supp}\left(I_{l}(f, u)(v)\right)=f(\operatorname{supp}(u[v]))$ correspondingly.

37. Definitions. Suppose that $Q$ is a category and $F, G$ are two functors in $Q$. Suppose also that a transformation $p: F \rightarrow G$ is defined for each $X \in Q$, that is a continuous mapping $p_{X}: F(X) \rightarrow G(X)$ is given. If $p_{Y} \circ F(f)=G(f) \circ p_{X}$ for each mapping $f \in \operatorname{Mor}(X, Y)$ and every objects $X, Y \in O b(Q)$, then the transformation $p=\left\{p_{X}: X \in O b(Q)\right\}$ is called natural.

If $T: Q \rightarrow Q$ is an endofunctor in a category $Q$ and there are natural transformations the identity $\eta: 1_{Q} \rightarrow T$ and the multiplication $\psi: T^{2} \rightarrow T$ satisfying the relations $\psi \circ T \eta=\psi \circ \eta T=1_{T}$ and $\psi \circ \psi T=\psi \circ T \psi$, then one says that the triple $\mathbf{T}:=(T, \eta, \psi)$ is a monad.

38. Theorem. There are monads in the categories $\mathcal{S} \times \mathcal{K}_{w}, \mathcal{S}_{l} \times \mathcal{K}, \mathcal{S} \times \mathcal{K}_{w, l}$ and $\mathcal{S}_{l} \times \mathcal{K}_{l}$.

Proof. Let $\bar{g}(v):=v(g)$ for $g \in C(X, K)$ and $v \in I(X, K)$, where $X \in O b(\mathcal{S})$ and $K \in O b\left(\mathcal{K}_{w}\right)$. Therefore, this induces the morphism $\bar{g}: I(X, K) \rightarrow K$. Then

$$
\begin{gathered}
\overline{g^{b} \times{ }_{2} g}(v)=v\left(g^{b} \times_{2} g\right)=b \times{ }_{2} v(g)=b \times 2 \bar{g}(v) \text { and } \\
\overline{g \times{ }_{2} g^{b}}(v)=v\left(g \times{ }_{2} g^{b}\right)=v(g) \times_{2} b=\bar{g}(v) \times_{2} b,
\end{gathered}
$$

where $g^{b}(x)=b$ for each $x \in X$, that is

(1) $\overline{g \times 2 g^{b}}=\bar{g} \times_{2} \overline{g^{b}}$ and $\left(1^{\prime}\right) \overline{g^{b} \times 2 g}=\overline{g^{b}} \times_{2} \bar{g}$

for each $g \in C(X, K)$ and $b \in K$.

Then we get $\overline{g \vee h}(v)=v(g \vee h)=v(g) \vee v(h)=\bar{g}(v) \vee \bar{h}(v)=(\bar{g} \vee \bar{h})(v)$. Moreover, we deduce that $\overline{g \wedge h}(v)=v(g \wedge h)=v(g) \wedge v(h)=\bar{g}(v) \wedge \bar{h}(v)=(\bar{g} \wedge \bar{h})(v)$. Thus we get the equalities

(2) $\overline{g \vee h}=\bar{g} \vee \bar{h}$ and $\left(2^{\prime}\right) \overline{g \wedge h}=\bar{g} \wedge \bar{h}$.

If additionally $v$ is left homogeneous and $K \in O b\left(\mathcal{K}_{w, l}\right)$, then $\overline{b g}=v(b g)=b v(g)=b \bar{g}(v)$. Therefore, we infer that $\overline{b g}=b \bar{g}$ for every $b \in K$ and $g \in C(X, K)$.

For $\lambda \in I(I(X, K), K)$ we put $\xi_{X, K}(\lambda)(g)=\lambda(\bar{g})$ for each $g \in C(X, K)$. Then $\xi_{X, K}(\lambda)\left(g^{b}\right)=$ $\lambda\left(\overline{g^{b}}\right)=\lambda\left(q^{b}\right)=b$, where $q^{b}: I(X, K) \rightarrow K$ denotes the constant mapping $q^{b}(y)=b$ for each $y \in I(X, K)$. From Formulas $\left(1,1^{\prime}\right)$ it follows that

$$
\begin{gathered}
\xi X, K \\
\xi_{X, K}(\lambda)\left(g g^{b} \times_{2} g\right)=\lambda\left(\overline{g^{b} \times_{2} g}\right)=\lambda\left(b \times_{2} \bar{g}\right)=\lambda\left(\overline{g \times 2 g^{b}}\right)=\lambda\left(\bar{g} \times{ }_{2} \overline{g^{b}}\right)=\lambda(\bar{g}) \times_{2} b=\xi_{X, K}(\lambda)(g) \times_{2} b .
\end{gathered}
$$

On the other hand, from Formulas $\left(2,2^{\prime}\right)$ we get that

$$
\begin{gathered}
\xi_{X, K}(v)(g \vee h)=v(\overline{g \vee h})=v(\bar{g} \vee \bar{h})=v(\bar{g}) \vee v(\bar{h})=\xi_{X, K}(v)(g) \vee \xi_{X, K}(v)(h) \text { and } \\
\xi_{X, K}(v)(g \wedge h)=v(\overline{g \wedge h})=v(\bar{g} \wedge \bar{h})=v(\bar{g}) \wedge v(\bar{h})=\xi_{X, K}(v)(g) \wedge \xi_{X, K}(v)(h)
\end{gathered}
$$

for each $b \in K, g, h \in C(X, K)$. Thus $\xi_{X, K}: I(I(X, K), K) \rightarrow I(X, K)$. 
If $\lambda \in I_{h}\left(I_{h}(X, K), K\right)$ for some $K \in O b\left(\mathcal{K}_{w, l}\right)$, then $\xi_{X, K}(\lambda)(b g)=\lambda(\overline{b g})=\lambda(b \bar{g})=b \lambda(\bar{g})$, hence $\xi_{X, K}: I_{h}\left(I_{h}(X, K), K\right) \rightarrow I_{h}(X, K)$. Analogously the mapping $\xi_{X, K}: \mathcal{O}(\mathcal{O}(X, K), K) \rightarrow \mathcal{O}(X, K)$ is defined for each $X \in O b(\mathcal{S})$ and $K \in \mathcal{K}_{w}$, also $\xi_{X, K}: \mathcal{O}_{l}\left(\mathcal{O}_{l}(X, K), K\right) \rightarrow \mathcal{O}_{l}(X, K), \xi_{X, K}:$ $I_{l}\left(I_{l}(X, K), K\right) \rightarrow I_{l}(X, K)$ for each $X \in O b\left(\mathcal{S}_{l}\right)$ and $K \in \mathcal{K}, \xi X, K: I_{h}\left(I_{h}(X, K), K\right) \rightarrow I_{h}(X, K)$ for $X \in O b(\mathcal{S})$ and $K \in \mathcal{K}_{w, l}, \xi_{X, K}: I_{l, h}\left(I_{l, h}(X, K), K\right) \rightarrow I_{l, h}(X, K)$ for $X \in O b\left(\mathcal{S}_{l}\right)$ and $K \in \mathcal{K}_{l}$. One also puts $\eta: I d_{Q} \rightarrow \mathcal{O}$ or $\eta: I d_{Q} \rightarrow I$ for $Q=\mathcal{S} \times \mathcal{K}_{w}$, also $\eta: I d_{Q} \rightarrow \mathcal{O}_{l}$ or $\eta: I d_{Q} \rightarrow I_{l}$ for $Q=\mathcal{S}_{l} \times \mathcal{K}$ correspondingly.

Next we verify that the transformations $\eta$ and $\xi$ are natural for each $f \in \operatorname{Mor}\left(X \times K_{1}, Y \times K_{2}\right)$, i.e. $f=(s, u), s \in \operatorname{Mor}(X, Y), u \in \operatorname{Mor}\left(K_{1}, K_{2}\right)$ :

$$
\begin{gathered}
\left.\eta_{\left(Y, K_{2}\right)} \circ \mathcal{O}((s, u))=\mathcal{O}\left(i d_{Y}, i d_{K_{2}}\right)\right) \circ \mathcal{O}((s, u)) \\
\left.=\mathcal{O}((s, u))=\mathcal{O}((s, u)) \circ \mathcal{O}\left(i d_{X}, i d_{K_{1}}\right)\right)=\mathcal{O}((s, u)) \circ \eta_{\left(X, K_{1}\right)} \\
\xi_{\left(Y, K_{2}\right)} \circ \mathcal{O}((s, u))\left[\mathcal{O}^{2}\left(X, K_{1}\right)\right]=\xi_{\left(Y, K_{2}\right)}\left(\mathcal{O}(\bar{s}, \bar{u})\left[\mathcal{O}\left(X, K_{1}\right)\right]\right) \\
\left.=\mathcal{O}((s, u)) \circ \eta_{\left(X, K_{1}\right)}\left[\mathcal{O}^{2}\left(X, K_{1}\right)\right]\right),
\end{gathered}
$$

where $\mathcal{O}^{m+1}(X, K):=\mathcal{O}\left(\mathcal{O}^{m}(X, K), K\right)$ for each natural number $m$ (see also $§ 20$ and Proposition 35).

For each $v \in \mathcal{O}(X, K)$ and $g \in C(X, K)$ one gets

$$
\begin{gathered}
\xi_{X, K} \circ \eta_{(\mathcal{O}(X, K), K)}(v)(g)=\eta_{(\mathcal{O}(X, K), K)}(v)(\bar{g})=\bar{g}(v)=v(g) \text { and } \\
\xi_{X, K} \circ \mathcal{O}\left(\eta_{(X, K)}\right)(v)(g)=\left(\mathcal{O}\left(\eta_{(X, K)}(v)\right)(\bar{g})=v\left(\bar{g} \circ \eta_{(X, K)}\right)=v(g) .\right.
\end{gathered}
$$

Let now $\tau \in \mathcal{O}^{3}(X, K)$ and $g \in C(X, K)$, then

$$
\begin{gathered}
\xi_{(X, K)} \circ \xi_{\mathcal{O}(X, K)}(\tau)(g)=\left(\xi_{\mathcal{O}(X, K)}(\tau)\right)(\bar{g})=\tau(\bar{g}) \text { and } \\
\xi_{(X, K)} \circ \mathcal{O}\left(\xi_{(X, K)}\right)(\tau)(g)=\left(\mathcal{O}\left(\xi_{(X, K)}\right)(\tau)\right)(\bar{g})=\tau\left(\bar{g} \circ \xi_{(X, K)}\right)=\tau(\bar{g}),
\end{gathered}
$$

where $\overline{\bar{g}} \in C\left(\mathcal{O}^{2}(X, K), K\right)$ is prescribed by the formula $(\overline{\bar{g}})(v)=v(\bar{g})$ for each $v \in \mathcal{O}^{2}(X, K)$. Thus $\mathbf{O}:=(\mathcal{O}, \eta, \xi)$ is the monad. Since $I$ is the restriction of the functor $\mathcal{O}$, the triple $\mathbf{I}:=(I, \eta, \xi)$ is the monad in the category $\mathcal{S} \times \mathcal{K}_{w}$ as well. Analogously $\mathbf{O}_{l}:=\left(\mathcal{O}_{l}, \eta, \xi\right)$ and $\mathbf{I}_{l}:=\left(I_{l}, \eta, \xi\right)$ form the monads in the category $\mathcal{S}_{l} \times \mathcal{K} ; \mathbf{O}_{h}=\left(\mathcal{O}_{h}, \eta, \xi\right)$ and $\mathbf{I}_{h}=\left(I_{h}, \eta, \xi\right)$ are the monads in $\mathcal{S} \times \mathcal{K}_{w, l}$; $\mathbf{O}_{l, h}=\left(\mathcal{O}_{l, h}, \eta, \xi\right)$ and $\mathbf{I}_{l, h}=\left(I_{l, h}, \eta, \xi\right)$ are the monads in $\mathcal{S}_{l} \times \mathcal{K}_{l}$.

39. Proposition. If a sequence

(1) $\ldots \rightarrow K_{n} \rightarrow K_{n+1} \rightarrow K_{n+2} \rightarrow \ldots$ in $\mathcal{K}_{w}$ (or in $\mathcal{K}$ ) is exact, then sequences

(2) $\ldots \rightarrow \mathcal{O}_{2}\left(X, K_{n}\right) \rightarrow \mathcal{O}_{2}\left(X, K_{n+1}\right) \rightarrow \mathcal{O}_{2}\left(X, K_{n+2}\right) \rightarrow \ldots$ and

(3) $\ldots \rightarrow I_{2}\left(X, K_{n}\right) \rightarrow I_{2}\left(X, K_{n+1}\right) \rightarrow I_{2}\left(X, K_{n+2}\right) \rightarrow \ldots$ are exact (analogously for $\mathcal{O}_{l, 2}$ and $I_{l, 2}$ correspondingly).

Proof. A sequence

$\ldots \rightarrow K_{n} \rightarrow K_{n+1} \rightarrow K_{n+2} \rightarrow \ldots$ is exact means that $s_{n}\left(K_{n}\right)=\operatorname{ker}\left(s_{n+1}\right)$ for each $n$, where $s_{n}: K_{n} \rightarrow K_{n+1}$ is an order-preserving continuous algebraic homomorphism, $k e r\left(s_{n+1}\right)=s_{n+1}^{-1}(0)$. Each continuous homomorphism $s_{n}$ induces the continuous homomorphism $\mathbf{s}_{n}: C\left(X, K_{n}\right) \rightarrow$ $C\left(X, K_{n+1}\right)$ point-wise $\left(\mathbf{s}_{n}(f)\right)(x)=s_{n}(f(x))$ for each $x \in X$. Therefore, we get that $\mathbf{s}_{n}(f \vee g)=$ $\mathbf{s}_{n}(f) \vee \mathbf{s}_{n}(g)$ or $\mathbf{s}_{n}(f \wedge g)=\mathbf{s}_{n}(f) \wedge \mathbf{s}_{n}(g)$, when $f \vee g$ or $f \wedge g$ exists, where $f, g \in C\left(X, K_{n}\right)$. Moreover, the equalities $\left(\mathbf{s}_{n}(f+g)\right)(x)=\mathbf{s}_{n}(f(x)+g(x))=s_{n}(f(x))+s_{n}(g(x))=\left[\mathbf{s}_{n}(f)+\right.$ $\left.\mathbf{s}_{n}(g)\right](x)$ and $\left[\mathbf{s}_{n}(f g)\right](x)=s_{n}(f(x) g(x))=s_{n}(f(x)) s_{n}(g(x))=\left[\left(\mathbf{s}_{n}(f)\right)\left(\mathbf{s}_{n}(g)\right)\right](x)$ are fulfilled, consequently, $\mathbf{s}_{n}\left(C\left(X, K_{n}\right)\right)=\mathbf{s}_{n+1}^{-1}(0)$, since $f_{n+2} \in C\left(X, K_{n+2}\right)$ is zero if and only if $f_{n+2}(x)=0$ for each $x \in X$. Thus the sequence

$$
\ldots \rightarrow C\left(X, K_{n}\right) \rightarrow C\left(X, K_{n+1}\right) \rightarrow C\left(X, K_{n+2}\right) \rightarrow \ldots \text { is exact. }
$$


Then a continuous morphism $\lambda_{n+2} \in \mathcal{O}\left(X, K_{n+2}\right)$ is zero on $\mathbf{s}_{n+1}\left(C\left(X, K_{n+1}\right)\right)$ if and only if $\lambda_{n+2}\left(f_{n+2}\right)=0$ for each $f_{n+2} \in \mathbf{s}_{n+1}\left(C\left(X, K_{n+1}\right)\right)$. Therefore, $\mathbf{s}_{n+1}\left(\lambda_{n+1}\right)=0=\lambda_{n+2}$ on $\mathbf{s}_{n+1}\left[\mathbf{s}_{n}\left(C\left(X, K_{n}\right)\right)\right]$ if and only if $\lambda_{n+1}\left(f_{n+1}\right) \in s_{n}\left(K_{n}\right)$ for each $f_{n+1} \in \mathbf{s}_{n}\left(C\left(X, K_{n}\right)\right)$. At the same time we have that $\mathbf{s}_{n+1}\left[\mathbf{s}_{n}\left(C\left(X, K_{n}\right)\right)\right] \subset \mathbf{s}_{n+1}\left(C\left(X, K_{n+1}\right)\right)$, consequently, $\mathcal{O}_{2}\left(s_{n}\right)=\operatorname{ker} \mathcal{O}_{2}\left(s_{n+1}\right)$. Thus the sequences $(2,3)$ are exact, analogously for other functors $I_{2}, \mathcal{O}_{l, 2}$ and $I_{2, l}$.

\subsection{Lattices Associated with Actions of Groupoids on Topological Spaces}

40. Lemma. Let $G$ be a topological groupoid with a unit acting on a topological space $X$ such that to each element $g \in G$ a continuous mapping $v_{g}: X \rightarrow X$ corresponds having the properties

(1) $v_{g} v_{h}=v_{g h}$ for each $g, h \in G$ and

(2) $v_{e}=i d$, where $e \in G$ is the unit element, id $(x)=x$ for each $x \in X$. If $K$ is a topological ringoid with the associative sub-ringoid $L, L \supset\{0,1\}$, such that

(3) $a(b c)=(a b) c$ for each $a, b \in L$ and $c \in K$, a continuous mapping $\rho: G^{2} \rightarrow L \backslash\{0\}$ satisfies the cocycle condition

(4) $\rho(g, x) \rho\left(h, v_{g} x\right)=\rho(g h, x)$ and

(5) $\rho(e, x)=1 \in K$ for each $g, h \in G$ and $x \in X$, then

(6) $T_{g} f(x):=\rho(g, x) \hat{v}_{g} f(x)$ is a representation of $G$ by continuous in the $g \in G$ variable mappings $T_{g}$ of $C(X, K)$ into $C(X, K)$, when $f$ is marked, where $f \in C(X, K), \hat{v}_{g} f(x):=f\left(v_{g}(x)\right)$ for each $g \in G$ and $x \in X$.

Proof. For each $g, h \in G$ one has $T_{g}\left(T_{h} f(x)\right)=\rho(g, x) \hat{v}_{g}\left[\rho(h, x) \hat{v}_{h} f(x)\right]=\rho(g h, x) \hat{v}_{g h} f(x)=$ $T_{g h} f(x)$, hence $T_{g} T_{h}=T_{g h}$. Moreover, $T_{e} f=f$, since $v_{e}=i d$ and $\rho(e, x)=1$, i.e., $T_{e}=I$ is the unit operator on $C(X, K)$. Mappings $T_{g} f(x)$ are continuous in the $g \in G$ variable as compositions and products of continuous mappings.

The continuous mappings $T_{g}$ are (may be) generally non-linear relative to $K$. If $K$ is commutative, distributive and associative, then $T_{g}$ are $K$-linear on $C(X, K)$.

41. Definition. A continuous morphism $v$ on $C(X, K)$ or $C_{+}(X, K)$ we call semi-idempotent, if it satisfies the property:

(1) $v(g+f)=v(g)+v(f)$ for each $f, g \in C(X, K)$ or $C_{+}(X, K)$ respectively, where $(g+f)(x)=$ $g(x)+f(x)$ for each $x \in X$.

Suppose that $G$ is a topological groupoid with the unit continuously acting on a topological space $X$ and satisfying Conditions $40(1,2)$. A continuous morphism $\lambda$ on $C(X, K)$ or $C_{+}(X, K)$ we call $(T, G)$-invariant if

(2) $\hat{T}_{g} \lambda=\lambda$, where $\left(\hat{T}_{g} \lambda\right)(f):=\lambda\left(T_{g} f\right)$ for each $g \in G$ and $f$ in $C(X, K)$ or $C_{+}(X, K)$ correspondingly.

Let $S_{+}(G, K)$ denote the family of all semi-idempotent continuous morphisms, when $K$ is commutative and associative relative to the addition for $(G, K)$, let also $S_{\vee}(G, K)$ (or $S_{\wedge}(G, K)$ ) denote the family of all continuous morphisms satisfying Conditions 2(4) (or 2(5) correspondingly) for general $K$. Denote by $H_{+}(G, K)$ (or $H_{\vee}(G, K)$ or $H_{\wedge}(G, K)$ ) the family of all $G$-invariant semi-idempotent (or in $S_{\vee}(G, K)$ or in $S_{\wedge}(G, K)$ correspondingly) continuous morphisms for $(X, K)$, when $X=G$ as a topological space. We supply these families with the operations of the addition

(3) $v(f)+{ }_{i} \lambda(f)=:\left(v+{ }_{i} \lambda\right)(f)$ in $S_{j}(G, K)$ for $i=1,2,3$ and $j=+, \vee, \wedge$ respectively and the multiplication being the convolution of continuous morphisms

(4) $(v * \lambda)(f)=v\left(\lambda\left(T_{g} f\right)\right)$ in $S_{j}(G, K)$, where $g \in G, j \in\{+, \vee, \wedge\}$.

Then we put $H_{h}(G, K), S_{h}(G, K), H_{\vee, h}(G, K), S_{\vee, h}(G, K), H_{\wedge, h}(G, K)$ and $S_{\wedge, h}(G, K)$ for the subsets of all left homogeneous morphisms in $H_{+}(G, K), S_{+}(G, K), H_{\vee}(G, K), S_{\vee}(G, K), H_{\wedge}(G, K)$, $S_{\wedge}(G, K)$ correspondingly. 
42. Proposition. If $v$ is a $(T, G)$-invariant semi-idempotent continuous morphism, then its support is contained in $\bigcap_{n=1}^{\infty} T^{n}(X)$, where

$$
T(A):=\bigcup_{g \in G} \operatorname{supp}\left(\rho(g, x) \hat{v}_{g}\left(\chi_{A}(x)\right)\right)
$$

for a closed subset $A$ in $X$. Moreover, if $K$ has not divisors of zero a support of $v$ is G-invariant and contained in $\bigcap_{n=1}^{\infty} P^{n}(X)$, where

$$
P(X)=\bigcup_{g \in G} v_{g}(X)
$$

Proof. If $v(f) \neq 0$, then $v\left(T_{g} f\right) \neq 0$ for each $g \in G$, when a continuous morphism $v$ is $(T, G)$-invariant. On the other hand, if $\operatorname{supp}(f) \subset \operatorname{supp}(v)$, then $\operatorname{supp}\left(\rho(g, x) \hat{v}_{g} f(x)\right) \subset \operatorname{supp}(v)$ for each $g \in G$. At the same time, $\bigcup_{g \in G} \operatorname{supp}\left(T_{g} f\right) \subset \bigcup_{g \in G} \operatorname{supp}\left(\hat{v}_{g} f\right)$, since $\rho(g, x) \in L \backslash\{0\}$ for each

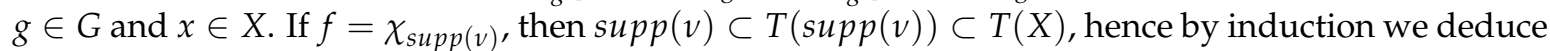
that $\operatorname{supp}(v) \subset T^{n}(X)$ for each natural number $n$, where $\chi_{A}$ is the characteristic function of a set $A$, so that $\chi_{A}(x)=1$ for each $x \in A$ while $\chi_{A}(x)=0$ for each $x \notin A$.

If $K$ has not divisors of zero, then $\operatorname{supp}\left(\hat{T}_{g} v\right)=\hat{v}_{g} \operatorname{supp}(v) \subset \operatorname{supp}(v)$ for each element $g \in G$, hence $\bigcup_{g \in G} \hat{v}_{g} \operatorname{supp}(v)=\operatorname{supp}(v)$, since $e \in G$ and $v_{e}=i d$. That is $\operatorname{supp}(v)$ is $G$-invariant. Since $\operatorname{supp}(v) \subset X$, then $\operatorname{supp}(v) \subset P(X)$ and by induction $\operatorname{supp}(v) \subset P^{n}(X)$ for each natural number $n$.

43. Proposition. If $G$ is a topological groupoid with a unit or a topological monoid, then $S_{+}(G, K)$, $S_{\vee}(G, K)$ and $S_{\wedge}(G, K)$ for general $T_{g}$ and $K$ (or $S_{h}(G, K), S_{h, \vee}(G, K)$ and $S_{h, \wedge}(G, K)$ for $T_{g} \equiv \hat{v}_{g}$ or when $K$ is commutative and associative relative to the multiplication) supplied with the convolution $41(4)$ as the multiplication operation are topological groupoids with a unit or monoids correspondingly.

Proof. Certainly, the definitions above imply the inclusion $S_{h}(G, K) \subset S_{+}(G, K)$. If $v, \lambda \in I_{h}(G, K)$, then $(v * \lambda)(b f)=v\left(\lambda\left(T_{g}(b f)\right)\right)=v\left(b \lambda\left(T_{g} f\right)\right)=b((v * \lambda)(f))$, when either $T_{g} \equiv \hat{v}_{g}$ for each $g \in G$ or $K$ is commutative and associative relative to the multiplication. We mention that the evaluation morphism $\delta_{e}$ at $e$ belongs to $S_{h}(G, K)$ and has the property $v * \delta_{e}=\delta_{e} * v=v$ for each $v \in S(G, K)$, where $e$ is a unit element in $G, \delta_{x} f=f(x)$ for each $f \in C(X, K)$ and $x \in X$. Thus $\delta_{e}$ is the neutral element in $S(G, K)$.

For a topological monoid $G$ one has $\hat{v}_{s}\left(\hat{v}_{u} f(x)\right)=f(s(u x))=f((s u) x)=\hat{v}_{s u} f(x)$ for each $f \in C(G, K)$ and $s, u, x \in G$ so that $f((s u) x)$ is a function continuous in the variables $s, u$ and $x$ in $G$. Since $v$ and $\lambda$ are continuous on $C(G, K)$, then $v * \lambda$ is continuous on $C(G, K)$.

If $G$ is a topological monoid, then $(v *(\lambda * \phi))(f)=v^{u}\left((\lambda * \phi)\left(T_{u} f\right)\right)=v^{u}\left(\lambda^{s}\left(\phi\left(T_{s} T_{u} f\right)\right)=\right.$ $v^{u}\left(\lambda^{s}\left(\phi\left(T_{s u} f\right)\right)\right)=(v * \lambda)^{s u}\left(\phi\left(T_{s u} f\right)\right)=[(v * \lambda) * \phi](f)$ for every $f \in C(G, K)$ and $u, s \in G$ and $v, \lambda, \phi \in S_{j}(G, K)$, where $v^{u}(h)$ means that a continuous morphism $v$ on a function $h$ acts by the variable $u \in G$, consequently, $v *(\lambda * \phi)=(\nu * \lambda) * \phi$. Thus the family $S_{j}(G, K)$ is associative, when $G$ is associative, where $j \in\{+, \vee, \wedge, h,(h, \vee),(h, \wedge)\}$ for the corresponding $T_{g}$ and $K$.

From $\S \S 2.3,2.4,2.9$ and 5 it follows that the mapping $(\nu, \lambda) \mapsto v * \lambda$ is continuous.

44. Theorem. If $G$ is a topological groupoid with a unit or a topological monoid, then $S_{+}(G, K)$ (for $K$ commutative and associative relative to +$), S_{\vee}(G, K)$ and $S_{\wedge}(G, K)$ for general $T_{g}$ (or $S_{\vee, h}(G, K)$ and $S_{\wedge, h}(G, K)$ for either $T_{g} \equiv \hat{v}_{g}$ or when $K$ is commutative and associative relative to the multiplication) are topological ringoids or semirings correspondingly.

Proof. If $f, g \in C(X, K)$ or in $C_{+}(X, K)$ and $f \vee g$ or $f \wedge g$ exists (see Condition (3) in Lemma 2.12), $v, \lambda$ are continuous morphisms satisfying Condition either 2(4) or 2(5) respectively, then

(1) $\left(v+{ }_{i} \lambda\right)\left(f+{ }_{i} g\right)=v\left(f+{ }_{i} g\right)+{ }_{i} \lambda\left(f+{ }_{i} g\right)=\left(v(f)+{ }_{i} v(g)\right)+{ }_{i}\left(\lambda(f)+{ }_{i} \lambda(g)\right)=\left(v(f)+{ }_{i}\right.$ $\lambda(f))+{ }_{i}\left(v(g)+{ }_{i} \lambda(g)\right)=\left(v+{ }_{i} \lambda\right)(f)+{ }_{i}\left(v+{ }_{i} \lambda\right)(g)$

for $i=1,2,3$, where $+_{1}=+,+_{2}=\vee,+_{3}=\wedge$. That is, the continuous morphism $v+{ }_{i} \lambda$ satisfies Property 41(1) for $i=1$ or 2(4) for $i=2$ or 2(5) when $i=3$ correspondingly. If additionally $v$ and $\lambda$ are left homogeneous, then

(2) $\left(v+{ }_{i} \lambda\right)(b f)=v(b f)+{ }_{i} \lambda(b f)=b v(f)+{ }_{i} b \lambda(f)=b\left(v+{ }_{i} \lambda\right)(f)$ for each $b \in K$. 
On the other hand, we deduce that

$$
\begin{aligned}
& \left(\left(v_{1}+{ }_{i} v_{2}\right) * \lambda\right)(f)=\left(v_{1}+{ }_{i} v_{2}\right)\left(\lambda\left(T_{g} f\right)\right)=v_{1}\left(\lambda\left(T_{g} f\right)\right)+{ }_{i} v_{2}\left(\lambda\left(T_{g} f\right)\right) \\
& =\left(v_{1} * \lambda\right)(f)+{ }_{i}\left(v_{2} * \lambda\right)(f) \text { and } \\
& \left(\lambda *\left(v_{1}+{ }_{i} v_{2}\right)\right)(f)=\lambda\left(\left(v_{1}+{ }_{i} v_{2}\right)\left(T_{g} f\right)\right)=\lambda\left(v_{1}\left(T_{g} f\right)\right)+{ }_{i} \lambda\left(v_{2}\left(T_{g} f\right)\right) \\
& =\left(\lambda * v_{1}\right)(f)+{ }_{i}\left(\lambda * v_{2}\right)(f)
\end{aligned}
$$

for each $v_{1}, v_{2}, \lambda \in S_{j}(G, K)$ and $f \in C(G, K)$ or in $C_{+}(G, K)$ correspondingly, for $i=1,2,3$ and $i=i(j)$ respectively, where $+_{1}=+,+_{2}=\vee$ and $+_{3}=\wedge$. Thus, the right and left distributive rules are satisfied:

(3) $\left(v_{1}+{ }_{i} v_{2}\right) * \lambda=v_{1} * \lambda+{ }_{i} v_{2} * \lambda$ and

(4) $\lambda *\left(v_{1}+{ }_{i} v_{2}\right)=\lambda * v_{1}+{ }_{i} \lambda * v_{2}$

for $i=1,2,3$ respectively. From the definitions of these operations and Proposition 43 their continuity follows.

Therefore, Formulas $(1-4)$ and Proposition 43 imply that $S_{+}(G, K), \quad S_{\vee}(G, K)$, $S_{\wedge}(G, K), \quad S_{\vee, h}(G, K)$ and $S_{\wedge, h}(G, K)$ are left and right distributive topological ringoids or semirings correspondingly.

45. Theorem. If $G$ is a topological groupoid with a unit, $X=G$ as a topological space (see §41), then $H_{j}(G, K)$ is a closed ideal in $S_{j}(G, K)$, where $j=+($ for $K$ commutative and associative relative to + ) or $j=\mathrm{V}$ or $j=\wedge$ or $j=(\vee, h)$ or $j=(\wedge, h)$ with $\rho(u, x) \equiv 1 ; j=(\vee, h)$ or $j=(\wedge, h)$ for commutative and associative $K$ relative to the multiplication with general $T_{u}$.

Proof. We mention that $\hat{T}_{g}\left(b_{1} \lambda_{1}+{ }_{i} b_{2} \lambda_{2}\right)(f)=b_{1} \lambda_{1}\left(T_{g} f\right)+{ }_{i} b_{2} \lambda_{2}\left(T_{g} f\right)$, where the operation denoted by the addition $+_{i}$ is either + or $\vee$ or $\wedge$ for $i=1$ or $i=2$ or $i=3$ correspondingly (and also below in this section), consequently, $b_{1} \lambda_{1}+{ }_{i} b_{2} \lambda_{2} \in H_{j}(G, K)$ for each $\lambda_{1}, \lambda_{2} \in H_{j}(G, K)$ and $b_{1}, b_{2} \in K, i=i(j)$.

In Formula 41(4) after the action of a morphism $\lambda$ on a continuous function $T_{g} f(x)$ in the variable $x$ one gets that $\lambda\left(T_{g} f\right)=: h(g)$ is a continuous function in the variable $g$ and $v$ is acting on this function, i.e. $(v * \lambda)(f)=v(h(x))$, where $x, g \in G$. This implies that

$$
\begin{aligned}
& v *\left(\lambda\left(f+{ }_{i} t\right)\right)=v *\left(\lambda(f)+{ }_{i} \lambda(t)\right)=v\left(\lambda\left(T_{g} f\right)+{ }_{i} \lambda\left(T_{g} t\right)\right) \\
& =v\left(\lambda\left(T_{g} f\right)\right)+{ }_{i} v\left(\lambda\left(T_{g} t\right)\right)=(v * \lambda)(f)+{ }_{i}(v * \lambda)(t) \text { for } i=1,2,3,
\end{aligned}
$$

consequently, the convolution operation maps from $S_{j}(G, K)^{2}$ into $S_{j}(G, K)$.

The property being $G$-invariant provides closed subsets in $S_{j}(G, K)$, since if a net of continuous mappings $g_{k}$ converges to a continuous mapping $g$ an each $g_{k}$ is $G$-invariant, then $g=\lim _{k} g_{k}$ is $G$-invariant as well.

If $\lambda \in H_{j}(G, K)$ and $v \in S_{j}(G, K)$, then

$\left(\hat{T}_{s}(v * \lambda)\right)(f)=\hat{T}_{s}\left(v^{u}\left(\lambda^{x}\left(T_{u} f(x)\right)\right)\right)=v^{u}\left(\lambda^{x}\left(T_{s}\left(T_{u} f(x)\right)\right)\right.$

$\left.=v^{u}\left(\lambda^{x}\left(T_{u} f(x)\right)\right)\right)=(v * \lambda)(f)$ and

$\left(\hat{T}_{s}(\lambda * v)\right)(f)=\hat{T}_{s}\left(\lambda^{u}\left(v^{x}\left(T_{u} f(x)\right)\right)\right)=\lambda^{u}\left(v^{x}\left(T_{s}\left(T_{u} f(x)\right)\right)\right)$

$=\lambda^{u}\left(T_{s}\left(v^{x}\left(T_{u} f(x)\right)\right)\right)=(\lambda * v)(f)$,

since $\lambda^{u}\left(T_{s} g(u)\right)=\lambda^{u}(g(u))=\lambda(g)$, particularly with $g(x)=T_{u} f(x)$ or $g(u)=v^{x}\left(T_{u} f(x)\right)$ correspondingly, whilst $T_{S} \equiv \hat{v}_{s}$ in the cases $j=+$ or $j=\vee$ or $j=\wedge$ with $\rho \equiv 1$, or for general $T_{u} f(x)=\rho(u, x) \hat{v}_{s} f(x)$ in the cases of homogeneous continuous morphisms $j=(\vee, h)$ or $j=(\wedge, h)$ (see $\S 43$ also), hence $v * \lambda, \lambda * v \in H_{j}(G, K)$. Therefore, the latter formula and Theorem 44 imply that

$$
\begin{aligned}
& \left(v+{ }_{i} H_{j}(G, K)\right) * H_{j}(G, K) \subset\left(v * H_{j}(G, K)\right)+{ }_{i}\left(H_{j}(G, K) * H_{j}(G, K)\right) \\
& \subset H_{j}(G, K)+{ }_{i} H_{j}(G, K) \subset H_{j}(G, K) \text { and } \\
& H_{j}(G, K) *\left(v++_{i} H_{j}(G, K)\right) \subset\left(H_{j}(G, K) * v\right)+{ }_{i}\left(H_{j}(G, K) * H_{j}(G, K)\right) \\
& \subset H_{j}(G, K)+{ }_{i} H_{j}(G, K) \subset H_{j}(G, K)
\end{aligned}
$$

for each $v \in S_{j}(G, K)$ and $+_{i}$ corresponding to $j$, that is $H_{j}(G, K)$ is the right and left closed ideal in $S_{j}(G, K)$. 


\section{Conclusions}

Skew continuous morphisms of ordered ringoids, semirings, algebroids and non-associative algebras can be used for studies of their structures and representations.

Conflicts of Interest: The author declares no conflict of interest.

\section{References}

1. Baez, J.C. The octonions. Bull. Am. Mathem. Soc. 2002, 39, 145-205.

2. Birkhoff, G. Lattice Theory; Mathematical Society: Providence, RI, USA, 1967.

3. Bourbaki, N. Algebra; Springer: Berlin, German, 1989.

4. Dickson, L.E. The Collected Mathematical Papers; Chelsea Publishing Co.: New York, NY, USA, 1975; Volumes 1-5.

5. Grätzer, G. General Lattice Theory; Akademie-Verlag: Berlin, German, 1978.

6. Kasch, F. Moduln und Ringe; Teubner: Stuttgart, German, 1977.

7. Kurosh, A.G. Lectures on General Algebra; Nauka: Moscow, Russian, 1973.

8. Schafer, R.D. An Introduction to nonassociative Algebras; Academic Press: New York, NY, USA, 1966.

9. Litvinov, G.L.; Maslov, V.P.; Shpiz. G.B. Idempotent functional analysis: an algebraic approach. Math. Notes 2001, 65, 696-729.

10. Ludkovsky, S.V. Topological transformation groups of manifolds over non-Archimedean fields, representations and quasi-invariant measures. J. Mathem. Sci. NY Springer 2008, 147, 6703-6846.

11. Ludkovsky, S.V. Topological transformation groups of manifolds over non-Archimedean fields, representations and quasi-invariant measures, II. J. Mathem. Sci., N. Springer 2008, 150, 2123-2223.

12. Ludkovsky, S.V. Operators on a non locally compact group algebra. Bull. Sci. Math. Paris Ser. 2 2013, 137, 557-573, doi:10.1016/j.bulsci.2012.11.008.

13. Ludkovsky, S.V. Meta-centralizers of non locally compact group algebras. Glasg. Mathem. J. 2015, 57, 349-364, doi:10.1017/S0017089514000330.

14. Weil, A. L'intégration Dans Les Groupes Topologiques et Ses Applications; Hermann: Paris, France, 1940.

15. Bucur, I.; Deleanu, A. Introduction to the Theory of Categories and Functors; Wiley: London, UK, 1968.

16. Fedorchuk, V.V. Covariant functors in the category of compacta, absolute retracts and $Q$-manifolds. Rissian Math. Surv. 1981, 36, 211-233.

17. Mitchell, B. Theory of Categories; Academic Press, Inc.: London, UK, 1965.

18. Shchepin, E.V. Functors and uncountable powers of compacta. Russ. Math. Surv. 1981, 36, 1-71.

19. Mendelson, E. Introduction to Mathematical Logic; D. van Nostrand Co., Inc.: Princeton, NJ, USA, 1964.

20. Kunen, K. Set Theory; North-Holland Publishing Co.: Amsterdam, Dutch, 1980.

21. Engelking, R. General Topology; Heldermann: Berlin, German, 1989.

22. Barwise, J. Ed. Handbook of Mathematical Logic; North-Holland Publishing Co.: Amsterdam, Dutch, 1977.

23. Ludkovsky, S.V. Properties of quasi-invariant measures on topological groups and associated algebras. Ann. Math. Blaise Pascal 1999, 6, 33-45.

(C) 2016 by the author; licensee MDPI, Basel, Switzerland. This article is an open access article distributed under the terms and conditions of the Creative Commons by Attribution (CC-BY) license (http:/ / creativecommons.org/licenses/by/4.0/). 